\title{
BIN1 regulates electrical activity and network synchronization in hiPSC-derived neurons
}

Orthis Saha ${ }^{1, \#}$, Ana Raquel Melo de Farias ${ }^{1,2, \#}$, Alexandre Pelletier ${ }^{3, \#}$, Arnaud Carrier ${ }^{3}$, Johanna Gadaut $^{1}$, Bruna Soares Landeira ${ }^{1}$, Karine Guyot ${ }^{1}$, Anaïs-Camille Vreulx ${ }^{1}$, Philippe Amouyel ${ }^{1}$, Amelie Bonnefond ${ }^{3}$, Cláudio Marcos Queiroz ${ }^{2}$, Devrim Kilinc ${ }^{1}$, Fabien Delahaye ${ }^{3}$, Jean-Charles Lambert ${ }^{1}$ and Marcos R. Costa ${ }^{1,2, *}$

1. Univ. Lille, Inserm, CHU Lille, Institut Pasteur de Lille, U1167-RID-AGE facteurs de risque et déterminants moléculaires des maladies liées au vieillissement, DISTALZ, Lille, France

2. Brain Institute, Federal University of Rio Grande do Norte, Natal, Brazil

3. Univ. Lille, Inserm, CNRS, CHU Lille, Institut Pasteur de Lille, U1283-UMR 8199 EGID, Lille, France

\# Equal contribution

* Correspondence should be addressed to:

Marcos Costa, MD, PhD

INSERM UMR1167

Institut Pasteur de Lille

1 rue du Pr. Calmette

59019 Lille CEDEX, France

Tel: 0033 (0)32087 7710

marcos.costa@pasteur-lille.fr 


\begin{abstract}
Alzheimer's disease (AD) is a multifactorial disease with a strong genetic background. Recent genomic wide association studies have identified several loci linked to an increased risk of $A D$. However, genes regulated by these variants and the pathophysiological mechanisms regulated by those genes remain largely elusive. In this work, we studied the role of Bridging Integrator 1 (BIN1), the second most important AD risk gene after APOE, in neurons generated from human induced pluripotent stem cells (hiPSCs) in bi-dimensional cultures and cerebral organoids. We show that deletion of BIN1 is sufficient to cause neuronal hyperactivation and network desynchronization in a cell-autonomous fashion. These functional changes are correlated with increased Tau phosphorylation and transcriptional changes similar to those observed in the brains of $A D$ patients, particularly in glutamatergic neurons. Together, our results reveal a role for BIN1 in the regulation of electrical activity in human neurons and suggest that its implication in $A D$ pathogenesis could be related to the neuronal and network dysfunctions observed in the AD brain.
\end{abstract}




\section{Introduction}

The Bridging Integrator 1 (BIN1) gene is the second most important genetic risk factor for late-onset Alzheimer's disease (LOAD), after the Apolipoprotein $E(A P O E)$ gene (Lambert et al., 2013; Kunkle et al., 2019; Bellenguez et al., 2020; Schwartzentruber et al., 2021). In the adult human brain, BIN1 is mainly expressed by oligodendrocytes, microglial cells, glutamatergic and GABAergic neurons (De Rossi et al., 2016, 2020; Marques-Coelho et al., 2021) and its expression is reduced in the brain of $A D$ patients compared to healthy individuals (Glennon et al., 2013; Leng et al., 2021; Marques-Coelho et al., 2021). Yet, how this reduced expression of BIN1 could affect AD pathogenesis remains poorly understood.

Besides the classical pathological markers observed in the brains of AD patients, such as accumulation of amyloid-beta $(A \beta)$ peptides in amyloid plaques, Tau hyperphosphorylation and aggregation in neurofibrillary tangles, microglial activation, synapse loss and neurodegeneration (Serrano-Pozo et al., 2011), increased neuronal electrical activity has recently emerged as an early phenotype in AD (Canter et al., 2016; Palop and Mucke, 2016; Vossel et al., 2017; Styr and Slutsky, 2018; Harris et al., 2020). Interestingly, accumulation of $A \beta$ peptides leads to neuronal hyperactivation (Busche et al., 2008, 2012; Gavello et al., 2018; Zott et al., 2019) and neuronal activity per se regulates the internalization of amyloidprecursor protein (APP) and production of $A \beta$ peptides (Cirrito et al., 2005, 2008; Das et al., 2013), suggesting the existence of a vicious cycle of $A \beta$-dependent neuronal hyperactivation that could contribute to neuronal dysfunctions at the onset of AD. Tauopathy also promotes neuronal network hyperexcitability (Busche et al., 2019) and hyperphosphorylated tau deposits were reported in epilepsy patients' brains (Vossel et al., 2017).

BIN1 may contribute to the regulation of neuronal and circuit activity through numerous mechanisms, including increased APP processing and production of amyloid-beta peptides (Miyagawa et al., 2016; Ubelmann et al., 2017), alterations in Tau phosphorylation and propagation (Calafate et al., 2016; Lasorsa et al., 2018; Sartori et al., 2019; Voskobiynyk et al., 2020), subcellular localization of voltage-gated calcium channels (Hong et al., 2010; Voskobiynyk et al., 2020) and recycling of synaptic vesicles/AMPA receptors (Schürmann et al., 2019; De Rossi et al., 2020). Accordingly, conditional deletion of BIN1 in neurons of the adult mice hippocampus, leads to altered frequency of mini excitatory post-synaptic currents 
(mEPSC), likely due to an impaired presynaptic release probability and slower depletion of neurotransmitters (De Rossi et al., 2020). Knockdown of BIN1 in embryonic rat primary cortical neurons also affects AMPA receptor trafficking in the post-synaptic compartment, leading to alterations in the amplitude of mini excitatory post-synaptic currents (mEPSC) (Schürmann et al., 2019). Lastly, overexpression of a BIN1-mKate2 fusion protein in embryonic rat hippocampal cultures leads to increased neuronal activity and frequency of spontaneous excitatory postsynaptic currents (sEPSCs), seemingly by affecting the localization of L-type voltage gated calcium channels in the membrane through a Tau-dependent interaction (Voskobiynyk et al., 2020).

Together, these evidence in animal models may suggest that BIN1 affects AD risk by regulating cellular processes directly or indirectly affecting neuronal electrical activity and synaptic transmission. Yet, the role of BIN1 in human neurons remains poorly understood. We have recently generated BIN1 knockout (KO) human induced pluripotent stem cells (hiPSCs) and shown that BIN1 neuronal-specific isoform 1 regulates endocytic trafficking in hiPSC-derived neurons (hiNs), a role conserved in human and drosophila neurons (Lambert et al., 2021). In this work, we characterized the alterations in neuronal electrical activity, network oscillations, synaptic connectivity and gene expression observed in hiNs following complete and partial BIN1 deletion. 


\section{Results}

\section{Transcriptional alterations in BIN1 KO hiNs target electrical activity and synaptic transmission}

To test whether specific deletion of BIN1 in hiNs could affect neuronal activity, we generated pure BIN1 WT or KO neuronal cultures by direct lineage-reprogramming of human Neural progenitor cells (hNPCS) using doxycycline-inducible expression of ASCL1 (Fig. 1A). We first confirmed that ASCL1 expression efficiently reprogrammed hiNPCs into highly pure neurons, hereafter named ASCL1-hiNs, before addition of exogenous human forebrain astrocytes (Fig. 1B-C). To support neuronal differentiation/maturation and synaptic connectivity, we added human cerebral cortex astrocytes in ASCL1-hiNs cultures following antibiotic selection (Fig. 1A) (Christopherson et al., 2005). This methodology allowed us to unambiguously probe the effects of BIN1 loss-of-function in neurons, since astrocytes in both WT and KO cell cultures have intact BIN1.

To assess the neuronal phenotypes in these cultures at the molecular level, we performed snRNA-seq experiments after 4 weeks of differentiation. At this stage, we observed that more than $70 \%$ of cells ( $n=3114$ from 2 independent culture batches) expressed pan-neuronal markers SOX11, SNAP25, DCX and RBFOX3, with $66 \%$ of cells co-expressing the glutamatergic neuron marker SLC17A6, less than $1.5 \%$ of cells co-expressing the GABAergic neuron markers $D L X 1, G A D 1$ and GAD2, and 5\% of cells co-expressing low levels of both neuronal subtype's markers (Fig. 1D-E). The remaining cells - immature astrocytes (Astro-I) and mature astrocytes (Astro-II) and undifferentiated NPCs, represented about 15\%, 8\%, 4\% of the cells, respectively (Fig. 1D). The first two cell types likely represent two different states of astrocytes added to the cultures, whereas NPCs are cells that failed to reprogram into hiNs despite ASCL1 transduction. Thus, ASCL1-hiNs are mainly glutamatergic neurons ( 92\%) with a small proportion of GABAergic neurons ( $2 \%)$ after 4 weeks of differentiation.

Next, we investigated possible transcriptional alterations in these different cell types using Wilcoxon test after SCTtransform normalization and variance stabilization of molecular count data (Hafemeister and Satija, 2019). We detected 675 differentially expressed genes (DEGs; $|\log 2 \mathrm{FC}|>0.25$ and FDR < 0.05) in BIN1 KO compared to WT glutamatergic neurons, 
and only 1 DEG in GABAergic neurons (Fig. 1E; Sup. Table 1). Consistent with the common origin of astrocytes in both BIN1 WT and KO hiN cultures, we observed a low number of DEGs in this cell type (25 in Astro-I and 18 in Astro-II; Sup. Table 1), likely reflecting an astrocyte reaction to primary changes in hiNs in response to BIN1 deletion. Gene ontology (GO) term enrichment analysis for DEGs identified in BIN1 KO hiNs revealed a significant enrichment for several terms associated with synaptic transmission and cation channel activity (Fig. 1G; Sup. Table 2), suggesting that BIN1-loss-of function leads to specific transcriptional changes associated with functional properties of neurons.

To investigate possible AD-like biochemical hallmarks in ASCL1-hiNs, we also measured phospho-TAU, full-length APP, APP CTF- $\beta$ and amyloid-beta (A $\beta$ ) protein/peptide in the extracellular medium by AlphaLISA and in the intracellular compartment by western blot. We detected a significant increase in the intracellular levels of phospho-TAU (Ser202, Thr205) in different ASCL1-induced BIN1 KO compared to WT hiNs (Sup. Fig. 1). In contrast, we could not detect any significant differences neither in the concentrations of soluble $A \beta(1-x) / A \beta(1-42)$ peptides, nor in the intracellular levels of full-length APP and APP CTF- $\beta$ in BIN1 KO compared to WT ASCL1-hiNs cultures (Sup. Fig. 1).

\section{BIN1 null deletion modifies electrical activity pattern in ASCL1-hiNs}

Altered BIN1 expression levels have previously been associated with changes in neuronal electrical activity and synaptic transmission in rodents (Schürmann et al., 2019; De Rossi et al., 2020; Voskobiynyk et al., 2020). To address whether loss of BIN1 in human neurons could have similar effects, we used multi-electrode arrays to record and quantify multi-unit activity (MUA) in ASCL1-hiNs cultures. To improve signal detection, we cultured cells in an original microfluidics device bound to MEA plates. Our cell culture chamber presents two main spaces, one suitable for both neurites and cell bodies (soma) and another where only axons could invade (Sup. Fig. 2). We found that electrodes located below microchannels into which only neuronal processes could grow showed high activity (presence of detected action potentials) compared to those electrodes located below cell soma (Sup. Fig. 2). Therefore, we concentrated our analysis to these channels exhibiting the highest electrical activity and restricted it to one row ( $n=16$ channels per cell culture) to avoid recording twice or more the same neuronal process. We observed a drastic difference in the temporal organization of 
MUA spikes, as evidenced at 4 weeks as an example (Fig. 2A). We also found that the mean number of MUAs significantly increased from 2 to 3 weeks of culture, reaching a plateau for both genotypes, with no significant changes in the numbers of detected spikes in BIN1 KO compared to WT ASCL1-hiNs at different timepoints (Fig. 2B-C). However, BIN1 null deletion in ASCL1-hiNs significantly increased the number of spike bursts at 4 weeks and a similar trend was observed at 6 weeks (Fig. 2D). In agreement with this electrophysiological data, we also observed a significant increase in the frequency of calcium transients in BIN1 KO compared to WT ASCL1-hiNs (Sup. Fig. 3). These calcium transients were significantly reduced by pharmacological blockage of the glutamate AMPA receptors using 6-cyano-7nitroquinoxaline-2,3-dione (CNQX) (Sup. Fig. 3; Sup. Movies 1 to 4). These observations indicate that BIN1 regulates neuronal electrical activity in a cell-autonomous fashion.

\section{Network desynchronization of ASCL1-hiNs circuits}

To further dissect the functional consequences of BIN1 deletion at the single-neuron resolution and investigate whether our previous observations in MUA could be explained by different number of active neurons in BIN1 WT and KO cultures, we used spike waveform clustering to isolate single-unit activity (SUA) from our MUA recordings (see Methods). We identified a similar number of neuronal components (SUA) contributing to signals identified by MUA between the two genotypes (WT: 4.9 2; KO: 5.27). Interestingly, we observed a significant reduction in the frequency (Fig. 3B) and an increase in the amplitude (Fig. 3C) of SUA in BIN1 KO compared to WT ASCL1-hiNs. We also found that the structure of firing patterns of single neurons identified by SUA (Fig. 3D) faithfully recapitulated the pattern observed by MUA analysis (Fig. 2A). Accordingly, both array wide spike detection rate (ASDR), which forms the basis of synchronized burst analyses, and cross-correlograms of SUAs using a bin window of $200 \mathrm{~ms}$ revealed striking modifications in the temporal structure of SUAs in BIN1 KO compared to WT ASCL1-hiNs (Fig. 3D). While neuronal activity of BIN1 WT neurons could be observed at periodic intervals of about 8-10s, the activity of BIN1 KO neurons did not show any oscillatory pattern (Fig. 3E-G). These data indicate that BIN1 deletion in neurons is sufficient to promote network desynchronization by altering the structure of firing patterns at the single neuronal level. 


\section{BIN1 WT and KO ASCL1-hiNs make similar numbers of synaptic contacts}

We next assessed whether the electrophysiological changes observed could be mediated at the synaptic connectivity level, with regards to the number and function of synapses. For this purpose, we quantified the number of synaptic contacts in ASCL1-hiNs cultivated in microfluidic chambers allowing the isolation of synaptic contacts identified by immunocytochemistry using antibodies against the presynaptic protein synaptophysin (SYP), post-synaptic protein HOMER and the neuronal microtubule protein MAP2 (Fig. 4A-C). Through confocal imaging and Z-stack acquisitions, we analyzed the SYP/HOMER colocalization on MAP2 surfaces (Kilinc et al., 2020). We did not find any significant difference in the number of synaptic spots in BIN1 KO compared to WT ASCL1-hiNs both at 4 and 6 weeks of differentiation (Fig. 4D), suggesting that synaptic connectivity was similar in both genotypes. Next, we functionally studied glutamatergic synapses using real-time imaging of ASCL1-hiNs expressing glutamate sensor iGLUSnFr (Marvin et al., 2018). Similar to our observations using immunocytochemistry, we could not detect differences neither in the number of active spots (putative glutamatergic synapses) nor in the frequency of events (changes in the fluorescence above the threshold) in BIN1 KO compared to WT ASCL1-hiNs (Fig. 4E; Sup. Movies 5 and 6). Thus, BIN1 deletion does not impair glutamatergic synaptic network in ASCL1-hiNs, suggesting that the network changes detected in BIN1 KO ASCL1-hiNs are likely a consequence of altered intrinsic properties.

BIN1 loss-of-function in cerebral organoids leads to transcriptional changes mainly in glutamatergic neurons

Cerebral organoids faithfully recapitulate fundamental aspects of the three-dimensional organization of the human brain, including the molecular specification of different neural cell types/subtypes and the generation of complex electrical activity patterns (Lancaster et al., 2013; Trujillo et al., 2019). To further investigate the potential role of BIN1 in the regulation of neuronal electrical activity and gene expression within this more holistic system, we generated cerebral organoids using isogenic BIN1 WT, KO and heterozygous (HET) hiPSCs (see Methods). After 6.5 months of culture, cerebral organoids were composed of all the major neural cell types identified by the expression of MAP2, GFAP and NESTIN and we did not 
observe any gross difference in size or morphology of cerebral organoids among the three genotypes (Fig. 5A). Western blot analyses confirmed the reduction and absence of BIN1 protein in BIN1 HET and KO cerebral organoids, respectively (Fig. 5B). We have then employed single-nucleus RNA sequencing (snRNA-seq) in 6.5 months-old BIN1 WT, HET and KO cerebral organoids ( $n=4$ for each genotype) to further characterize cell types/subtypes in this system. After quality control, we recovered 4398 nuclei that could be grouped into 7 major cell clusters based on the expression of cell type markers SLC1A3 (GLAST), GFAP and TNC (astrocyte); SNAP25, DCX, MAPT (pan-neuronal); SLC17A7 (VGLUT1) and SLC17A6 (glutamatergic neurons); DLX1 and GAD2 (GABAergic neurons); HES6, CCND2 and CDK6 (NPCs); ITGA8 (choroid plexus); and CLIC6 (pigmented epithelium) (Fig. 5C-D). As previously shown for the human brain, BIN1 expression in cerebral organoids was mainly detected in oligodendrocytes and glutamatergic neurons (Fig. 5D). We observed a slight reduction and increase, respectively, in the proportion of glutamatergic neurons and astrocytes in BIN1 HET and KO compared to WT (Fig. 5E).

To examine potential DEGs in BIN1 KO or HET compared to WT cells, we performed Wilcoxon test after SCTtransform normalization for each major cell type identified in cerebral organoids. Consistent with the predominant expression of BIN1 in glutamatergic neurons in our cerebral organoids (Fig. 5D), we identified a high number of DEGs in this cell type both in BIN1 HET (76 genes) and KO (124 genes) compared to WT genotype (Fig. 5F). We also detected 75 DEGs in BIN1 KO, but only 6 DEGs in BIN1 HET compared to WT astrocytes (Fig. 5F). For all other cell types, we observed a maximum of 1-4 DEGs in the comparison between KO vs WT or HET vs WT (Sup. Table 3). These observations suggest that BIN1 loss-of-function mainly affects glutamatergic neurons, in a dose-dependent manner. Accordingly, we observed a significant overlap between DEGs identified in BIN1 HET and KO glutamatergic neurons (Fig. 5G). GO term enrichment analysis for DEGs identified in BIN1 KO or HET revealed a significant enrichment for several terms associated with synaptic transmission (Fig. 5H). For BIN1 KO glutamatergic neurons, we also identified GO terms associated with calcium binding and ion channels (Sup. Table 4), suggesting that BIN1-loss-of function leads to specific transcriptional changes associated with functional properties of this in this neuronal subtype. 
bioRxiv preprint doi: https://doi.org/10.1101/2022.01.18.476601; this version posted January 20, 2022. The copyright holder for this preprint (which was not certified by peer review) is the author/funder, who has granted bioRxiv a license to display the preprint in perpetuity. It is made available under aCC-BY-NC-ND 4.0 International license.

\section{Evidence of altered neuronal electrical activity in BIN1 HET and KO cerebral organoids}

We next assessed whether possible alterations in neuronal electrical activity could be detected in a more complex model susceptible to mimic brain organization. For this purpose, we performed electrophysiological recordings in 5-6 months-old samples of BIN1 HET and KO cerebral organoids, using a 256-channels multi-electrode array (Fig. 6A). We observed a significant increase in the frequency of detected spikes in BIN1 KO compared to WT cerebral organoids, but no significant changes were observed in BIN1 HET cerebral organoids (Fig. 6C). However, both the amplitude and frequency of spikes detected in cerebral organoids were much lower than those observed in our microfluidics/MEA 2D cultures (Fig. 2 and 3), which might hamper the detection of subtle alterations in the electrical activity.

To circumvent this limitation, we used an indirect strategy to evaluate changes in neuronal electrical activity within cerebral organoids by using single cell RNAseq data generated from WT, HET et KO BIN1 organoids. Indeed, several groups have shown that electrical activity promotes the expression of activity-related genes (ARGs) in neurons (Flavell and Greenberg, 2008) and that ARG-signatures can be used to retrospectively infer neuronal activity (Tyssowski et al., 2018). While neurons stimulated with brief patterns of electrical activity transcribe rapid primary response genes (rPRGs) or early response genes (ERGs), those stimulated with sustained patterns of electrical activity express delayed primary response genes (dPRGs), secondary response genes (SRGs) and late response genes (LRGs) (Hrvatin et al., 2018; Tyssowski et al., 2018). Using CellID (Cortal et al., 2021), we analyzed the enrichment for these gene signatures (Sup. Table 5) in our cerebral organoids at single-cell resolution. As expected, we observed that ARGs signatures were predominantly enriched in GABAergic and glutamatergic neurons, supporting the validity of this approach (Fig. 6D; Sup. Fig.4A). Quantification of the proportion of neurons significantly enriched for specific signatures $\left(p_{a d j}<0.05\right)$ revealed a significantly higher proportion of BIN1 HET and KO compared to WT glutamatergic neurons enriched for dPRGs, as well as an increased proportion of glutamatergic neurons enriched for SRGs and LRGs in BIN1 KO compared to WT (Fig. 6E; Sup. Fig.4B). Conversely, both BIN1 WT glutamatergic and GABAergic neurons showed a higher proportion of cells enriched for rPRGs compared to both genotypes (Fig. 6D-E). Thus, partial and complete deletion of BIN1 is associated with molecular signatures of sustained electrical activity specifically in glutamatergic neurons. 


\section{Molecular alterations in BIN1 KO cerebral organoids resemble those observed in the brains of $A D$ patients}

Using a snRNA-seq dataset generated from the entorhinal cortex (EC) and superior frontal gyrus (SFG) of AD patients at different Braak stages (Leng et al., 2021), we observed a progressive and significant decline of BIN1 mRNA levels in glutamatergic neurons (Fig. 7A), suggesting that reduced BIN1 expression could be a common feature during AD pathology progression. These observations prompt us to ask whether transcriptomic alterations observed in our BIN1 HET or KO cells in 2D and cerebral organoids, potentially related to electrical activity dysregulation in glutamatergic neurons, could also be observed in an AD pathophysiological context. To address this possibility, we independently compared DEGs identified in glutamatergic neurons and astrocytes in our cell culture models with those identified in the same cell types in AD brains (Sup. Table 6). Interestingly, DEGs identified in BIN1 HET or KO glutamatergic neurons and in BIN1 KO astrocytes showed a statistically significant overlap with DEGs detected in these cell populations of the AD brain at different Braak stages (Fig. 7B). This correlation was particularly strong between DEGs identified in glutamatergic neurons when comparing in BIN1 WT vs KO cultures and Braak 6 vs 0 glutamatergic neurons in the superior frontal gyrus of AD patients (Fig. 7B). GO analysis using as input the DEGs overlapping between glutamatergic neurons of BIN1 ASCL1 hiNs and AD brains are significantly enriched for pathways associated with glutamate receptor activity and gated channel activity (Fig. 7C). Similarly, the overlap of DEGs in glutamatergic neurons of BIN1 KO cerebral organoids and those of AD brains are significantly enriched for genes associated with glutamate receptor activity, gated channel activity and calcium ion binding (Fig. 7D; Sup. Table 7). No significant enrichment could be observed for common DEGs in astrocytes of BIN1 KO cerebral organoids and AD brains (data not shown). Altogether, these observations suggest that BIN1 loss-of-function is sufficient to elicit gene expression alterations in glutamatergic neurons resembling those observed in AD brains and associated with regulation of neuronal electrical properties. 


\section{Discussion}

In this work, we show that BIN1, the second most important risk factor for AD after APOE, plays a critical role in the regulation of neuronal firing homeostasis. Complete deletion of BIN1 gene in glutamatergic neurons is sufficient to alter the electrical activity of these cells and abolish oscillatory network activity in human neuron circuits in vitro. These functional changes are correlated with changes in the expression of genes involved in synaptic transmission and ion transport across the membrane, as well as increased Tau phosphorylation. In long term neuronal cultures using cerebral organoids, we confirm that BIN1 loss-of-function affects electrical activity and leads to transcriptional and biochemical alterations resembling those observed in the $A D$ brain. These results suggest that misexpression of BIN1 in glutamatergic neurons may contribute to early stages of $A D$ pathophysiology by dysregulating neuronal firing homeostasis, which in turn would lead to other cellular and molecular alterations resulting in circuit dysfunctions and cognitive decline.

Neuronal network dysfunctions are observed in AD patients at early stages of the disease and precede or coincide with cognitive decline (Vossel et al., 2013, 2017; Lam et al., 2017). Under physiological conditions, neuronal networks can maintain optimal output through regulation of synaptic and cell-intrinsic mechanisms (Turrigiano and Nelson, 2004). Our results suggest that normal levels of BIN1 expression in glutamatergic neurons are fundamental to regulate neuronal firing rate homeostasis. Indeed, BIN1 deletion in hiNs is sufficient to enhance the amplitude of spikes, increase spike burst frequency and dysregulate network oscillations without changes in the number of functional synaptic contacts. This could suggest that the desynchronization observed in BIN1 KO hiNs circuits are a consequence of miscarried homeostatic controls of neuronal activity at early stages.

One key mechanism controlling neuronal spiking activity is the regulation of $\mathrm{Ca}^{2+}$ homeostasis. Increased neuronal electrical activity induces the turnover of L-type VGCC from the neuronal surface through endocytosis (Green et al., 2007) and regulates the transcription of genes encoding for calcium-binding proteins and calcium-mediated signaling (Dörrbaum et al., 2020), both mechanisms aiming to restore local $\mathrm{Ca}^{2+}$ signaling cascades and protect cells against inappropriate global $\mathrm{Ca}^{2+}$ signals. BIN1 interacts and regulates the localization of Ltype VGCC in cardiac T tubules (Hong et al., 2010). Intriguingly, in mouse hippocampal 
neurons, BIN1 interacts with L-type VGCC in a Tau-dependent manner and regulates neuronal electrical activity (Voskobiynyk et al., 2020). It is thus tempting to speculate that altered neuronal and network activity in BIN1 KO and HET hiNs could be at least partly explained by changes in the localization of neuronal L-type VGCC. Consistent with this idea, pharmacological blockage of these channels alters the synchronous firing patterns of hiPSCderived neural networks (Plumbly et al., 2019).

Loss of $\mathrm{Ca}^{2+}$ homeostasis is an important feature of many neurological diseases and has been extensively described in AD (Bezprozvanny and Mattson, 2008; Carvalho et al., 2021). Interestingly, DEGs identified in glutamatergic neurons in our different cell culture models are enriched for calcium-related biological processes. This is also observed for DEGs commonly detected in glutamatergic neurons of BIN1 KO organoids and AD brains. Thus, reduced expression of BIN1 in glutamatergic neurons may contribute to the breakdown of $\mathrm{Ca}^{2+}$ homeostasis in the $A D$ brain, potentially contributing to neuronal circuit dysfunctions. Consistent with this possibility, we have previously shown a significant reduction in the expression of the transcript encoding for the neuronal-specific BIN1 isoform 1 in bulk RNAsequencing data from a large number of AD patients (Marques-Coelho et al., 2021).

Future studies should evaluate whether variants in the BIN1 locus associated with an increased AD susceptibility could regulate BIN1 RNA splicing. It has already been shown that the deletion of a $363 \mathrm{bp}$ region containing some of these variants affects BIN1 expression in hiPSC-derived microglial cells (Alexi et al., 2019). An insertion/deletion variant has also been shown to modulate transcriptional activity in neuroblastoma (Chapuis et al., 2013). Unfortunately, the effect of this variant (rs59335482) in the expression of BIN1 in hiPSCderived cells could not be directly addressed due to the impossibility to perform CRISPR/Cas9gene editing in this region (variant is an expansion/contraction of $\mathrm{G}$ nucleotides, which means that the protospacer adjacent motif sequence will remain after the gene editing has occurred, and consequently, allow re-cutting and INDEL formation after repair). Yet, we may postulate that individuals carrying such variant could have subtle alterations in the expression of BIN1 in glutamatergic neurons, which throughout life undermine $\mathrm{Ca}^{2+}$ homeostasis in these cells but are not sufficient to provoke circuit-level dysfunction before senectitude.

Besides calcium homeostasis, BIN1 has also been suggested to regulate neuronal electrical activity and synaptic transmission through the regulation of recycling of synaptic vesicles/AMPA receptors (Schürmann et al., 2019; De Rossi et al., 2020). Although we did not 
observe any significant difference in the number and function of glutamatergic synapses in BIN1 KO hiNs, we cannot completely rule out the possibility that the same processes are affected in these cells. Together with the well-known roles of L-type VGCC in modulating neuronal gene transcription and synaptic plasticity (Deisseroth et al., 2003), these observations highlight BIN1 as a central player of neural network activity. Future studies should address whether individuals carrying genomic variants in the BIN1 locus associated with AD risk are more likely to show electroencephalographic alterations (Palop and Mucke, 2016; Vossel et al., 2017) and, therefore, could benefit of prophylactic treatments aiming at restoring network activity. 


\section{Acknowledgements and financial support}

The authors thank the BICeL platform of the Institut Biologie de Lille and the Vect'UB viral platform (INSERM US 005 - CNRS 3427 - TBMCore, Université de Bordeaux, France). The authors thank Karine Blary at the IEMN Lille for the microfabrication work. This work was cofunded by the European Union under the European Regional Development Fund (ERDF) and by the Hauts de France Regional Council (contract no.18006176), the MEL (contract_2016_ESR_05), and the French State (contract no. 2018-3-CTRL_IPL_Phase2). This work was partly supported by the French RENATECH network (P-18-02737), Fondation pour la recherche médicale (ALZ201912009628, ALZ201906008477) and by the Sanofi i-Awards Europe 2019. This work was also funded by the Lille Métropole Communauté Urbaine and the French government's LABEX DISTALZ program (Development of innovative strategies for a transdisciplinary approach to Alzheimer's disease). The UMR 8199 LIGAN-PM Genomics platform (Lille, France) belongs to the 'Federation de Recherche' 3508 Labex EGID (European Genomics Institute for Diabetes; ANR-10-LABX-46) and was supported by the ANR Equipex 2010 session (ANR-10-EQPX-07-01; 'LIGAN-PM'). The LIGAN-PM Genomics platform (Lille, France) is also supported by the FEDER and the Region Nord-Pas-de-Calais-Picardie and is a member of the "France Génomique" consortium (ANR-10-INBS-009).

\section{Declaration of interests}

The authors declare no competing interests.

\section{Author contributions}

Conceptualization, M.R.C., J.C.L, F.D.; Methodology, M.R.C., F.D., D.K.; Investigation, M.R.C., O.S., B.S.L., A.R.M.F., A.C., A.P., F.D., K.G., D.K., C.M.Q.; Writing - Original Draft, M.R.C.; Writing - Reviews \& Editing, M.R.C., F.D., J.C.L., A.P., O.S.; Figures preparation: M.R.C., F.D., A.P. Supervision, M.R.C., F.D., J.C.L., D.K.; Funding Acquisition, M.R.C., J.C.L., F.D, D.K. All authors have read and approved the manuscript. 


\section{References}

Alexi $\mathrm{N}$ et al. (2019) Brain cell type-specific enhancer-promoter interactome maps and disease-risk association. Science (80- ) 366:1134-1139 Available at: https://doi.org/10.1126/science.aay0793.

Bellenguez C, Küçükali F, Jansen I, Andrade V, Moreno-grau S (2020) New insights on the genetic etiology of Alzheimer's and related dementia. :1-35.

Bezprozvanny I, Mattson MP (2008) Neuronal calcium mishandling and the pathogenesis of

Alzheimer's disease. Trends Neurosci 31:454-463 Available at: https://doi.org/10.1016/j.tins.2008.06.005.

Busche MA, Chen X, Henning HA, Reichwald J, Staufenbiel M, Sakmann B, Konnerth A (2012) Critical role of soluble amyloid- $\beta$ for early hippocampal hyperactivity in a mouse model of Alzheimer's disease. Proc Natl Acad Sci U S A 109:8740-8745.

Busche MA, Eichhoff G, Adelsberger H, Abramowski D, Wiederhold K, Haass C, Staufenbiel M, Konnerth A, Garaschuk O (2008) Model of Alzheimer's Disease. :1686-1690.

Busche MA, Wegmann S, Dujardin S, Commins C, Schiantarelli J, Klickstein N, Kamath T V., Carlson GA, Nelken I, Hyman BT (2019) Tau impairs neural circuits, dominating amyloid$\beta$ effects, in Alzheimer models in vivo. Nat Neurosci 22:57-64 Available at: http://dx.doi.org/10.1038/s41593-018-0289-8.

Calafate S, Flavin W, Verstreken P, Moechars D (2016) Loss of Bin1 Promotes the Propagation of Tau Pathology. Cell Rep 17:931-940.

Canter RG, Penney J, Tsai LH (2016) The road to restoring neural circuits for the treatment of Alzheimer's disease. Nature 539:187-196.

Carvalho LI, Lambert J-C, Costa MR (2021) Analysis of modular gene co-expression networks reveals molecular pathways underlying Alzheimer's disease and progressive supranuclear palsy. medRxiv:2021.09.21.21263793 Available at: http://medrxiv.org/content/early/2021/10/05/2021.09.21.21263793.1.abstract.

Chapuis J et al. (2013) Increased expression of BIN1 mediates Alzheimer genetic risk by modulating tau pathology. Mol Psychiatry 18:1225-1234.

Christopherson KS, Ullian EM, Stokes CCA, Mullowney CE, Hell JW, Agah A, Lawler J, Mosher DF, Bornstein P, Barres BA (2005) Thrombospondins are astrocyte-secreted proteins that promote CNS synaptogenesis. Cell 120:421-433. 
Cirrito JR, Kang JE, Lee J, Stewart FR, Verges DK, Silverio LM, Bu G, Mennerick S, Holtzman DM (2008) Endocytosis Is Required for Synaptic Activity-Dependent Release of Amyloid- $\beta$ In Vivo. Neuron 58:42-51.

Cirrito JR, Yamada KA, Finn MB, Sloviter RS, Bales KR, May PC, Schoepp DD, Paul SM, Mennerick S, Holtzman DM (2005) Synaptic activity regulates interstitial fluid amyloid- $\beta$ levels in vivo. Neuron 48:913-922.

Cortal A, Martignetti L, Six E, Rausell A (2021) Gene signature extraction and cell identity recognition at the single-cell level with Cell-ID. Nat Biotechnol.

Das U, Scott DA, Ganguly A, Koo EH, Tang Y, Roy S (2013) Activity-induced convergence of app and bace-1 in acidic microdomains via an endocytosis-dependent pathway. Neuron 79:447-460.

De Rossi P, Buggia-Prévot V, Clayton BLL, Vasquez JB, Van Sanford C, Andrew RJ, Lesnick R, Botté A, Deyts C, Salem S, Rao E, Rice RC, Parent A, Kar S, Popko B, Pytel P, Estus S, Thinakaran G (2016) Predominant expression of Alzheimer's disease-associated BIN1 in mature oligodendrocytes and localization to white matter tracts. Mol Neurodegener 11. De Rossi P, Nomura T, Andrew RJ, Masse NY, Sampathkumar V, Musial TF, Sudwarts A, Recupero AJ, Le Metayer T, Hansen MT, Shim HN, Krause S V., Freedman DJ, Bindokas VP, Kasthuri N, Nicholson DA, Contractor A, Thinakaran G (2020) Neuronal BIN1 Regulates Presynaptic Neurotransmitter Release and Memory Consolidation. Cell Rep 30:3520-3535.e7 Available at: https://doi.org/10.1016/j.celrep.2020.02.026.

Deisseroth K, Mermelstein PG, Xia H, Tsien RW (2003) Signaling from synapse to nucleus: the logic behind the mechanisms. Curr Opin Neurobiol 13:354-365 Available at: https://www.sciencedirect.com/science/article/pii/S095943880300076X.

Dörrbaum AR, Alvarez-Castelao B, Nassim-Assir B, Langer JD, Schuman EM (2020) Proteome dynamics during homeostatic scaling in cultured neurons. Elife 9.

Flavell SW, Greenberg ME (2008) Signaling Mechanisms Linking Neuronal Activity to Gene Expression and Plasticity of the Nervous System. Annu Rev Neurosci 31:563-590 Available at: https://doi.org/10.1146/annurev.neuro.31.060407.125631.

Gavello D, Calorio C, Franchino C, Cesano F, Carabelli V, Carbone E, Marcantoni A (2018) Early Alterations of Hippocampal Neuronal Firing Induced by Abeta42. Cereb Cortex 28:433446.

Glennon EBC, Whitehouse IJ, Miners JS, Kehoe PG, Love S, Kellett KAB, Hooper NM (2013) 
BIN1 Is Decreased in Sporadic but Not Familial Alzheimer's Disease or in Aging. PLoS One 8:1-11.

Green EM, Barrett CF, Bultynck G, Shamah SM, Dolmetsch RE (2007) The Tumor Suppressor elF3e Mediates Calcium-Dependent Internalization of the L-Type Calcium Channel CaV1.2. Neuron 55:615-632.

Hafemeister C, Satija R (2019) Normalization and variance stabilization of single-cell RNA-seq data using regularized negative binomial regression. bioRxiv:1-15.

Harris SS, Wolf F, De Strooper B, Busche MA (2020) Tipping the Scales: Peptide-Dependent Dysregulation of Neural Circuit Dynamics in Alzheimer's Disease. Neuron:1-19 Available at: https://doi.org/10.1016/j.neuron.2020.06.005.

Hong TT, Smyth JW, Gao D, Chu KY, Vogan JM, Fong TS, Jensen BC, Colecraft HM, Shaw RM (2010) BIN1 localizes the L-type calcium channel to cardiac T-tubules. PLoS Biol 8.

Hrvatin S, Hochbaum DR, Nagy MA, Cicconet M, Robertson K, Cheadle L, Zilionis R, Ratner A, Borges-Monroy R, Klein AM, Sabatini BL, Greenberg ME (2018) Single-cell analysis of experience-dependent transcriptomic states in the mouse visual cortex. Nat Neurosci 21:120-129 Available at: https://doi.org/10.1038/s41593-017-0029-5.

Kilinc D, Vreulx A-C, Mendes T, Flaig A, Marques-Coelho D, Verschoore M, Demiautte F, Amouyel P, Eysert F, Dourlen P, Chapuis J, Costa MR, Malmanche N, Checler F, Lambert J-C (2020) Pyk2 overexpression in postsynaptic neurons blocks amyloid $\beta 1$-42-induced synaptotoxicity in microfluidic co-cultures. Brain Commun:1-18.

Kunkle BW et al. (2019) Genetic meta-analysis of diagnosed Alzheimer's disease identifies new risk loci and implicates $A \beta$, tau, immunity and lipid processing. Nat Genet 51:414430.

Lam AD, Deck G, Goldman A, Eskandar EN, Noebels J, Cole AJ (2017) Silent hippocampal seizures and spikes identified by foramen ovale electrodes in Alzheimer's disease. Nat Med 23:678-680 Available at: https://doi.org/10.1038/nm.4330.

Lambert JC et al. (2013) Meta-analysis of 74,046 individuals identifies 11 new susceptibility loci for Alzheimer's disease. Nat Genet 45:1452-1458.

Lancaster MA, Renner M, Martin CA, Wenzel D, Bicknell LS, Hurles ME, Homfray T, Penninger JM, Jackson AP, Knoblich JA (2013) Cerebral organoids model human brain development and microcephaly. Nature 501:373-379 Available at: http://dx.doi.org/10.1038/nature12517. 
Lasorsa A, Malki I, Cantrelle FX, Merzougui H, Boll E, Lambert JC, Landrieu I (2018) Structural basis of tau interaction with BIN1 and regulation by tau phosphorylation. Front Mol Neurosci 11:1-12.

Leng K, Li E, Eser R, Piergies A, Sit R, Tan M, Neff N, Li SH, Rodriguez RD, Suemoto CK, Leite REP, Ehrenberg AJ, Pasqualucci CA, Seeley WW, Spina S, Heinsen H, Grinberg LT, Kampmann M (2021) Molecular characterization of selectively vulnerable neurons in Alzheimer's disease. Nat Neurosci 24:276-287 Available at: http://dx.doi.org/10.1038/s41593-020-00764-7.

Marques-Coelho D et al. (2021) Differential transcript usage unravels gene expression alterations in Alzheimer's disease human brains. npj Aging Mech Dis 7:2 Available at: https://doi.org/10.1038/s41514-020-00052-5.

Marvin JS et al. (2018) Stability, affinity, and chromatic variants of the glutamate sensor iGluSnFR. Nat Methods 15:936-939.

Miyagawa T, Ebinuma I, Morohashi Y, Hori Y, Chang MY, Hattori H, Maehara T, Yokoshima S, Fukuyama T, Tsuji S, Iwatsubo T, Prendergast GC, Tomita T (2016) BIN1 regulates BACE1 intracellular trafficking and amyloid- $\beta$ production. Hum Mol Genet 25:2948-2958.

Palop JJ, Mucke L (2016) Network abnormalities and interneuron dysfunction in Alzheimer disease. Nat Rev Neurosci 17:777-792.

Plumbly W, Brandon N, Deeb TZ, Hall J, Harwood AJ (2019) L-type voltage-gated calcium channel regulation of in vitro human cortical neuronal networks. Sci Rep 9.

Sartori M et al. (2019) BIN1 recovers tauopathy-induced long-term memory deficits in mice and interacts with Tau through Thr348 phosphorylation. Acta Neuropathol 138:631-652 Available at: https://doi.org/10.1007/s00401-019-02017-9.

Schürmann B, Bermingham DP, Kopeikina KJ, Myczek K, Yoon S, Horan KE, Kelly CJ, Martinde-Saavedra MD, Forrest MP, Fawcett-Patel JM, Smith KR, Gao R, Bach A, Burette AC, Rappoport JZ, Weinberg RJ, Martina M, Penzes P (2019) A novel role for the late-onset Alzheimer's disease (LOAD)-associated protein Bin1 in regulating postsynaptic trafficking and glutamatergic signaling. Mol Psychiatry.

Schwartzentruber J, Cooper S, Liu JZ, Barrio-Hernandez I, Bello E, Kumasaka N, Young AMH, Franklin RJM, Johnson T, Estrada K, Gaffney DJ, Beltrao P, Bassett A (2021) Genome-wide meta-analysis, fine-mapping and integrative prioritization implicate new Alzheimer's disease risk genes. Nat Genet 53:392-402 Available at: 
http://dx.doi.org/10.1038/s41588-020-00776-w.

Serrano-Pozo A, Frosch MP, Masliah E, Hyman BT (2011) Neuropathological alterations in Alzheimer disease. Cold Spring Harb Perspect Med 1:1-24.

Styr B, Slutsky I (2018) Imbalance between firing homeostasis and synaptic plasticity drives early-phase Alzheimer's disease. Nat Neurosci 21:463-473.

Trujillo CA, Gao R, Negraes PD, Gu J, Buchanan J, Preissl S, Wang A, Wu W, Haddad GG, Chaim IA, Domissy A, Vandenberghe M, Devor A, Yeo GW, Voytek B, Muotri AR (2019) Complex Oscillatory Waves Emerging from Cortical Organoids Model Early Human Brain Network Development. Cell Stem Cell 25:558-569.e7 Available at: https://doi.org/10.1016/j.stem.2019.08.002.

Turrigiano GG, Nelson SB (2004) Homeostatic plasticity in the developing nervous system. Nat Rev Neurosci 5:97-107.

Tyssowski KM, DeStefino NR, Cho JH, Dunn CJ, Poston RG, Carty CE, Jones RD, Chang SM, Romeo P, Wurzelmann MK, Ward JM, Andermann ML, Saha RN, Dudek SM, Gray JM (2018) Different Neuronal Activity Patterns Induce Different Gene Expression Programs. Neuron 98:530-546.e11 Available at: https://doi.org/10.1016/j.neuron.2018.04.001.

Ubelmann F, Burrinha T, Salavessa L, Gomes R, Ferreira C, Moreno N, Guimas Almeida C (2017) Bin1 and CD 2 AP polarise the endocytic generation of beta-amyloid . EMBO Rep 18:102-122.

Voskobiynyk Y, Roth JR, Cochran JN, Rush T, Carullo NVN, Mesina JS, Waqas M, Vollmer RM, Day JJ, McMahon LL, Roberson ED (2020) Alzheimer's disease risk gene BIN1 induces Taudependent network hyperexcitability. Elife 9:1-25.

Vossel KA, Beagle AJ, Rabinovici GD, Shu H, Lee SE, Naasan G, Hegde M, Cornes SB, Henry ML, Nelson AB, Seeley WW, Geschwind MD, Gorno-Tempini ML, Shih T, Kirsch HE, Garcia PA, Miller BL, Mucke L (2013) Seizures and Epileptiform Activity in the Early Stages of Alzheimer Disease. JAMA Neurol 70:1158-1166 Available at: https://doi.org/10.1001/jamaneurol.2013.136.

Vossel KA, Tartaglia MC, Nygaard HB, Zeman AZ, Miller BL (2017) Review Epileptic activity in Alzheimer's disease: causes and clinical relevance. Available at: www.thelancet.com/neurology.

Zott B, Simon MM, Hong W, Unger F, Chen-Engerer HJ, Frosch MP, Sakmann B, Walsh DM, Konnerth A (2019) A vicious cycle of $\beta$ amyloid-dependent neuronal hyperactivation. 
bioRxiv preprint doi: $\mathrm{https}$ ://doi.org/10.1101/2022.01.18.476601; this version posted January 20, 2022. The copyright holder for this preprint (which was not certified by peer review) is the author/funder, who has granted bioRxiv a license to display the preprint in perpetuity. It is made available under aCC-BY-NC-ND 4.0 International license.

Science (80- ) 365:559-565. 


\section{Figure legends}

Figure 1: Generation of enriched glutamatergic hiNs using ASCL1. (A) Schematics showing the main steps involved in the generation of ASCL1-hiNs. (B) Images showing BIN1 WT and KO hiNs 7 days after the beginning of doxycycline treatment immunolabeled for the neuronal markers MAP2/TUBB3 and stained with DAPI. In this experiment, no astrocytes were added. C) UMAP representation of the different subpopulations in ASCL1-hiNs. D) Dot plot representing expression of key markers used to annotate cell populations. E) Volcano plot representing DEG comparing BIN1 KO vs WT in glutamatergic Neurons. Differentially expressed genes with adjusted $p$-value $<0.05$ and $|\log 2 \mathrm{FC}|>0.25$ are shown in red. Gene label is shown for top genes. F) Functional enrichment analysis in the DEGs of the Glutamatergic neurons. Dot plot representing the top10 enriched GO terms in each category at adjusted pvalue $<0.01$

Figure 2: Increased spike burst frequency in BIN1 KO ASCL-hiNs. (A) Raster plots showing MUA recorded for 1 minute in BIN1 WT and KO ASCL1-hiNs after 4 weeks of differentiation. Each line represents one electrode localized side-by-side in our microfluidic/MEA array (see Sup. Fig. 2). (B-C) Quantification of the number of detected spikes at different time points ( ${ }^{*}$ Padj= $0.0141 ; * * *$ Padj $=0.0006$; Two-way ANOVA followed by Tukey's multiple-comparison test; $n=5$ for each genotype). (D) Quantification of the number of spike bursts at different time points ( ${ }^{*} \mathrm{P}=0.004 ; \# \mathrm{P}=0.0888$; Mann-Whitney test).

Figure 3: Disorganization of neuron spike patterns in BIN1 KO ASCL1-hiNs. (A) Schematics showing the decomposition of MUA (black lines) into SUA (colored lines) using spike waveform clustering. (B) Quantification of single-neuron spikes or SUA (*** P=0.0004; MannWhitney test; $n=5$ for each genotype). (C) Quantification of SUA amplitudes ( ${ }^{*} P=0.0106$; Mann-Whitney test). (D) Raster plots showing SUA recorded from 5 different electrodes of BIN1 WT (left) or KO (right) ASCL-hiNs cultures after 4 weeks of differentiation. (E) Array-wide spike detection rate (ASDR) plots based on SUA recorded in BIN1 WT and KO ASCL1-hiNs cultures. Each line represents one independent culture batch. (F-G) Normalized crosscorrelogram heatmap (F) and normalized correlation (G) for all SUAs recorded in 5 independent BIN1 WT and KO ASCL1-hiNs cultures. 
Figure 4: Similar synaptic density and function in BIN1 WT and KO ASCL1-hiNs. (A) Schematics showing the design of microfluidic device used to isolate pre-synaptic (pre), post-synaptic (post) and synaptic (syn) compartments. (B-C) Immunocytochemistry using the astrocyte marker GFAP, neuronal marker MAP2, pre-synaptic marker HOMER and post-synaptic marker SYP in BIN1 WT ASCL1-hiNs culture after 4 weeks of differentiation. Rectangular box in B is magnified in C, allowing the identification of putative synaptic contacts ( $\left.C^{\prime}\right)$. (D) Quantification of SYP spots assigned to HOMER spots in MAP2 processes at 4 and 6 weeks ASCL1-hiNs cultures ( $n=8$ for each genotype). (E) Quantification of active spots per neuron and number of events detected by time-lapse video-microscopy in 4 or 6 weeks ASCL1-hiNs cultures transduced with the glutamate sensor iGLUSnFr ( $n=3$ for each genotype).

Figure 5: Cellular composition and transcriptional changes in BIN1 WT, HET and KO cerebral organoids. (A) Immunohistochemistry detecting GFAP+ astrocytes (red), MAP2+ neurons (green) and DAPI+ nuclei (blue) in 6.5 months old BIN1 WT, HET and KO cerebral organoids. (B) Western blot analysis for BIN1 showing the reduction and absence of protein in HET and KO cerebral organoids, respectively. C) UMAP representation of the different subpopulations in cerebral organoids. D) Dot plot representing expression for BIN1 and key markers used to annotate cell populations. E) Cell proportions in each subpopulation in the 3 genotypes $(* * * * P<0.0001$; Chi-square test). F) Volcano plot representing DEG comparing HET vs WT and KO vs WT in astrocytes and glutamatergic neurons. Differentially expressed genes with adjusted $p$-value $<0.05$ and $|\log 2 \mathrm{FC}|>0.25$ are shown in red. Gene label are shown for top10 gene in term of log2FoldChange and of $p$-value $G$ ) Venn diagram representing overlap in DEG between KO vs WT and HET vs WT H) Functional enrichment analysis in Glutamatergic neurons DEGs. Dot plot representing the top10 enriched GO terms in each category at adjusted pvalue $<0.01$. Percentage of DEG corresponds to the percentage of DEG overlapping with the total number of DEGs

Figure 6: Altered electrical activity in BIN1 HET and KO cerebral organoids. (A) Representative raster plot showing detected spikes in 5.5 months old BIN1 WT, HET and KO cerebral organoids recorded in a multi-well MEA device. (B) Quantification of active channels (>6 spikes/min) in 3-4 independent cerebral organoids of each genotype. (C) Quantification of the spike frequency in $\mathrm{Hz}$ ( ${ }^{*} \mathrm{Padj}=0.0206 ;{ }^{*} \mathrm{Padj}=0.0034$; Kruskal-Wallis followed by Dunn's 
multiple comparisons test). (D) Feature plots showing the enrichment score of single cells for ARG signatures. Enrichment scores correspond to the -log10(adjusted p-value) of the CellID based enrichment test (E) Quantification of the proportion of glutamatergic (left) and GABAergic neurons (right) enriched for the different ARG signatures according to genotypes $\left({ }^{*} p<0.05 ;{ }^{* *} p<0.001\right.$; Chi-Squared test). rPRGs: rapid primary response genes; dPRGs: delayed primary response genes; SRGs: secondary response genes (Tyssowski et al., 2018)

Figure 7: Similar transcriptional alterations in BIN1 mutated hiNs and glutamatergic neurons of the AD brain. (A) Boxplot representing BIN1 mRNA in expression through different Braak stages in entorhinal cortex (EC) and superior frontal gyrus (SFG) (***Padj<0.001; Wilcoxon test). (B) Dot plot representing the overlap between DEGs identified in glutamatergic neurons of the AD brain and BIN1 KO ASCL1-hiN cultures (left), BIN1 HET cerebral organoids (center) or BIN1 KO cerebral organoids (right). (C-D) Network representation of enriched GO terms in overlapping DEGs between AD brains and BIN1 mutated glutamatergic neurons. Enriched GO terms were identified using over-representation test. (C) GO enrichment in genes overlapping between DEGs identified in ASCL1-hiNs cultures comparing KO vs WT and DEGs identified in SFG comparing Braak stage 6 vs 0. (D) GO enrichment in genes overlapping between DEGs identified in Cerebral organoid comparing KO vs WT and DEGs identified in EC comparing Braak stage 6 vs 0 . Edges represents the genes membership to a GO term. Overlapping genes associated to the different pathways are annotated. 


\section{Supplementary data}

Sup. Figure 1: Altered Tau phosphorylation in BIN1 KO ASCL1-hiNs. (A) Western blots showing the expression of TAU-C, p-TAU (AT8) and B-ACTIN in 4 weeks old ASCL1 hiNs cultures. (B) Quantification of the ratios TAU-C/ACTIN, $\mathrm{p}-\mathrm{TAU} / \mathrm{B}-\mathrm{ACTIN}$ and $\mathrm{p}-\mathrm{TAU} / \mathrm{TAU}-\mathrm{C}\left({ }^{*} \mathrm{p}<0.05\right.$; Unpaired t-test; $n=7$ for each genotype). (C) Western blots showing the expression of APP full-length, CTF-ß and ß-ACTIN. (D) Quantification of the ratios APP/ß-ACTIN, CTF-ß/ß-ACTIN and CTF-ß/APP ( $n=5$ for each genotype). (E) Quantification of $A ß_{1-x}, A ß_{1-42}$ and the ratio $A ß_{1-}$ 42/ $A ß_{1-x}$ in ASCL1-hiNs cultures at 3 and 4 weeks.

Sup. Figure 2: Microfluidic/MEA device for in vitro electrophysiology. (A) Bright-field image of ASCL1-hiNs cultures on microfluidic/MEA devices showing the cell chamber and micro channels. Observe that neuron somas are mainly restricted to the cell chamber, whereas neuronal processes occupy micro channels. (B) Representative raster plots of electrophysiological recordings in the cell chamber and micro channels showing the higher sensitivity of the latter.

Sup. Figure 3: Altered frequency of calcium transients in BIN1 KO ASCL1-hiNs. (A) Snapshot of a 4 weeks-old ASCL1-hiNs culture labeled with Oregon green BAPTA. (B-B') Representative plot of fluorescence change over time in 1000 frames. Green vertical lines indicate detected calcium spikes (deltaF > 2 SDs). Dashed box is magnified in $B^{\prime}$. (C) Quantification of calcium spikes in BIN1 WT and KO ASCL1-hiNs before (-) and after (+) treatment with CNQX (*Padj= $0.0419 ;{ }^{* * P a d j}=0.0060 ;{ }^{* * *}$ Padj<0.0001; Kruskal-Wallis followed by Dunn's multiple comparison test; $n=3$ independent cultures for each genotype; number of active cells per condition: 754 (WT), 41 (WT + CNQX); 1006 (KO); 38 (KO + CNQX)).

Sup. Figure 4: Increased expression of late response genes in BIN KO cerebral organoids. (A) Dimension plots showing the enrichment score of single cells for ARG signatures. (B) Quantification of the proportion of glutamatergic (left) and GABAergic neurons (right) enriched for the different ARG signatures according to genotypes (Chi-Squared test; ***: p<0.001). ERGs: early response genes; LRGs: late response genes; Exc: excitatory neurons; Inh: inhibitory neurons (Hrvatin et al., 2018). 
Sup. Movies 1 to 4: Time-series of 1000 frames taken from BIN1 WT and KO ASCL1-hiNs after 4 weeks of differentiation and labeled with Oregon Green BAPTA and imaged. Videos are played at 100 frames per second (fps).

Sup. Movies 5 and 6: Time-series of 1000 frames taken from BIN1 WT and KO ASCL1-hiNs transduced with iGLUSnFr after 2 weeks of differentiation and imaged 2 weeks later. Videos are played at $100 \mathrm{fps}$.

Sup. Table 1: DEGs identified in different cell types/subtypes of ASCL1-hiNs cultures.

Sup. Table 2: GO terms enriched for DEGs identified in different cell types/subtypes of ASCL1hiNs cultures.

Sup. Table 3: DEGs identified in different cell types/subtypes of cerebral organoids.

Sup. Table 4: GO terms enriched for DEGs identified in different cell types/subtypes of cerebral organoids.

Sup. Table 5: List of ARGs used for Multiple Correspondence Analysis (MCA) in CellID.

Sup. Table 6: DEGs identified in different cell types/subtypes of the AD brain.

Sup. Table 7: GO terms enriched for DEGs commonly identified in BIN1 HET or KO cells and the AD brain. 


\section{Materials and methods}

\section{Maintenance of cells and generation of hiNPCs and hiNs}

hiPSCs modified for BIN1 in exon3 by CRISPR/Cas9 technology were sourced from Applied StemCell Inc. CA, USA. In addition to the BIN1 WT and KO hiPSCS, heterozygous (HET) iPSCS, harbouring a 1 bp insertion in one allele were also sourced Applied Stem Cells Inc. CA, USA.The parental cell line used for derivation of the cells was ASE 9109. The maintenance of these cells and the generation of hiNS, hiAs, and cerebral organoids thereof, have been detailed in the publication by Lambert et al., 2022. All hiPSCs and their neuronal and glial cell derivatives including cerebral organoids were maintained in media from Stemcell Technologies, Vancouver, Canada. Maintenance of cell cultures and cerebral organoids were done following manufacturer's protocols which have been elucidated on the webpage of Stemcell Technologies. In addition, the embryoid body method detailed by Stemcell Technologies was used for the induction of BIN1 WT and KO hiPSCs. Cell numbers and viability were recorded using a LUNA ${ }^{\mathrm{TM}}$ Automated Cell Counter (Logos Biosystems, South Korea).

hiNs generated from ASCL1-transduced hiNPCs (protocol detailed in next section) were subjected to differentiation for 4 weeks. All differentiations were performed in tissue in 24well cell imaging plates (0030741005, Eppendorf) culture dishes pre-coated with Poly-Lornithine (P4957, Sigma-Aldrich) and Mouse Laminin (CC095, Sigma-Aldrich).

\section{Differentiation protocol for induced hiNPCs}

We differentiated neurons from virus-transduced hiNPCs according to an adapted protocol (Zhang et al., 2013; Yang et al., 2017). Briefly, hiNPCs are first transfected with the TTA lentiviral construct and a passage later, the TetO-Ascl1-Puro lentiviral construct was transduced. These cells are maintained in NPM medium and expanded prior to differentiation. For differentiation of hiNs, hiNPCs are plated onto PLO/laminin-coated imaging plates at density 100,000 cells/well in NPM. After $24 \mathrm{~h}$, complete BrainPhys medium (BP) is added 1:1 together with $2 \mu \mathrm{g} / \mathrm{mL}$ doxycycline (Sigma-Aldrich) to induce TetO gene expression. The following day, $1 \mu \mathrm{g} / \mathrm{mL}$ puromycin (Sigma-Aldrich) was added to start cell selection. After 2-3 days (depending on the efficiency of antibiotic selection), 50,000 human cortical astrocytes were added in each well with BrainPhys containing doxycycline. After 24 hours, $2 \mu \mathrm{M}$ of Ara-C (Cytosine $\beta$-D-arabinofuranoside) (Sigma-Aldrich) was added to arrest 
the proliferation of astrocytes. Half of the medium in each well was changed biweekly with fresh BrainPhys medium (StemCell Technologies) containing doxycycline until the 14th day. After that, the biweekly medium change was performed only with BrainPhys. Differentiation was allowed to continue for another 2 weeks prior to subjecting the cells to various experimental manipulations.

Human cortical astrocytes (Catalog \# 1800) were sourced from ScienCell Research Laboratories, CA, USA. Maintenance and proliferation of astrocytes were done as per specifications mentioned on the datasheet from the provider which is available on their webpage.

\section{Culture of Induced Neurons (hiNs) in Microfluidic Devices}

Preparation of Microfluidic Devices: Three-compartment microfluidic neuron culture devices were used in which the presynaptic and postsynaptic chambers are connected to the synaptic chamber by respectively long and short micro-channels. Details of the microfluidic device design and fabrication have been previously described (Kilinc et al, 2020).

The homemade devices were placed individually in Petri dishes for easy handling and UV sterilized for $30 \mathrm{~min}$ before coating for cell adhesion. The primary surface coating consisted of poly-L-lysine (Sigma-Aldrich) at $20 \mu \mathrm{g} / \mathrm{mL}$ in borate buffer ( $0.31 \%$ boric acid, $0.475 \%$ sodium tetraborate, $\mathrm{pH} 8.5$ ). All coated devices were incubated overnight at $37^{\circ} \mathrm{C}, 5 \% \mathrm{CO}$. After a wash with DPBS, devices were then coated with $20 \mu \mathrm{g} / \mathrm{mL}$ laminin in DPBS and still incubated overnight at $37^{\circ} \mathrm{C}$ in $5 \% \mathrm{CO} 2$. The following day, devices were carefully washed once with DPBS before cells were plated onto them.

Cell Culture: In total, 30,000 NPCs prepared in complete Neural Progenitor Medium (NPM, Stemcell Technologies) containing $10 \mu \mathrm{M}$ of Y-27632 ROCK Inhibitor were seeded per device, half at the entrance of the presynaptic somatic chamber and half at the entrance of the postsynaptic somatic chamber. Microfluidic devices were microscopically checked at the phase contrast in order to ensure the cells were correctly flowing into chambers. After a minimum of 5 minutes to allow the cells to attach, devices were filled with NPM (containing $10 \mu \mathrm{M}$ of Y-27632 ROCK Inhibitor). Water was added to the Petri dishes to prevent media evaporation and these were then incubated at $37^{\circ} \mathrm{C}$ in a humidified $5 \% \mathrm{CO} 2$ incubator. The spontaneous neuronal differentiation of NPCs started 24 hours later and was done by performing half-medium change with complete BrainPhys Neuronal Medium. Induced 
neurons cultures were maintained during 4 to 6 weeks with biweekly change of half volume of BrainPhys medium.

For induced neurons culture from NPCs transduced for Ascl1, doxycycline $(2 \mu \mathrm{g} / \mathrm{mL})$ was added on the first day of half-medium change in order to induce TetO gene expression. The following day, puromycin $(1 \mu \mathrm{g} / \mathrm{mL})$ was added to start cell selection. Two days after the puromycin selection, a total of 5,000 human cortical astrocytes (ScienCell Research Laboratories, CA, USA) were added per device. After 24 hours, Ara-C ( $2 \mu \mathrm{M})$ was added to stop their proliferation. Half of the medium was changed twice a week with complete BrainPhys medium $+2 \mu \mathrm{g} / \mathrm{mL}$ doxycycline until 14 days. After that, half medium change was performed only with BrainPhys medium.

Four microfluidic devices were employed for each experimental condition (BIN1 KO versus WT in both spontaneous neuronal differentiation and Ascl1 induced one) and two independent cultures were performed. To assess the time-course effect, neuron cultures were stopped at 4 and 6 weeks.

\section{Generation of Cerebral Organoids}

Cerebral organoids (3D Cultures) were generated from wild-type, heterozygous and knockout hiPSCs using a 4-stage protocol (Lancaster et al., 2013). The first step was the Embryoid Body (EB) Formation Stage, where hiPCSc at 80\% confluency were detached from the Vitronectin XF substrate using Gentle Cell Dissociation Reagent (Stem Cell Technologies). To form the EB, 9000 cells were plated per well in a 96-well round-bottom ultra-low attachment plate containing EB seeding medium (Stem Cell Technologies). After two days, the EBs were transferred to a 24-well ultra-low attachment plate containing Induction Medium (Stem Cell Technologies), where each well receives 1-2 EBs. This was the Induction Stage. Two days later, the EBs were ready for the Expansion Stage. The EBs were embedded in Matrigel (Corning) and transferred to a 24-well ultra-low adherent plate with Expansion Medium (Stem Cell Technologies). After three days, the medium was replaced by Maturation Medium (Stem Cell Technologies) and the plate was placed in an orbital shaker (100 rpm speed). During this final Maturation Phase, full media change was done on a biweekly basis. Organoids were allowed to mature for a period of 6 months. 


\section{Viral Transductions}

Lentiviral constructs were produced by the Vect'UB platform within the TBM Core unit at University of Bordeaux, Bordeaux, France (CNRS UMS 3427, INSERM US 005). The lentiviral constructs used were TTA (ID \# 571) and TetO-Ascl1-Puro (Addgene, Plasmid \# 97329). Lentiviral infections were done in NPCs at P3 or P4. The viral constructs were transduced at a multiplicity of infection (MOI) of 2.5. In brief, NPCs were plated at a confluency of 1x106 cells per well of a 6 -well plate. After 4 hours of plating the cells, appropriate volumes of each lentiviral construct were mixed in complete Neural Progenitor medium and $50 \mu$ of the viral medium mix was then added to each well. We transduced the TTA construct at first in the NPCs. Following one passage, the TTA-transduced cells were transduced with the construct for Ascl1. Cells having both viral constructs were then further expanded for 1 or 2 passages before being used for differentiation into hiNs.

The iGluSnFR construct was an adeno-associated viral vector (BS11-COG-AAV8) sourced from Vigene Biosciences, MD, USA. The viral construct was transduced at a MOI of 5,000 at around 10 days of differentiation for the ASCL1-hiNs. Differentiation was allowed to continue for a duration of 4 weeks prior to imaging.

\section{Immunocytochemistry and Immunohistochemistry}

Bidimensional (2D) cultures: All cells were fixed in 4\% (w/v) paraformaldehyde (Electron Microscopy Sciences, Catalog \# 15712) for 10 minutes in the imaging plates. Following, fixation, cells were washed thrice with PBS 0.1 M. Blocking solution ( $5 \%$ normal donkey serum $+0.1 \%$ Triton X-100 in PBS $0.1 \mathrm{M}$ ) was added to fixed cells at room temperature for 1 hour under shaking conditions. After the blocking step, primary antibodies were added to cells in the blocking solution and incubated overnight at $4^{\circ} \mathrm{C}$. The following day, cells were washed

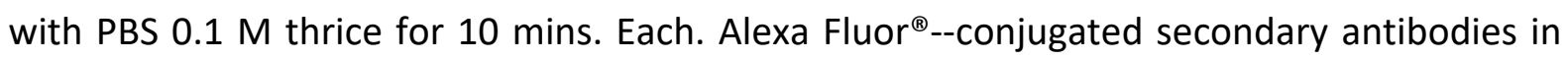
blocking solution were then incubated with the cells for 2 hours at room temperature under shaking conditions ensuring protection from light. Subsequently, 3 washes with 0.1 M PBS were done for 10 mins. each at room temperature under shaking conditions with protection from light. Hoechst 33258 solution was added during the second PBS wash. Cells were mounted with Aqua-Poly/Mount (Polysciences, Inc.) and imaged directly in the cell imaging plates. All images were acquired using an LSM 880 Confocal Scanning Microscope housed at the Imaging Platform of the Pasteur Institute, Lille. 
Antibodies used for immunocytochemistry were: MAP2 (188006, Synaptic Systems), Beta III Tubulin (MAB1637, Sigma-Aldrich), GFAP (AB5804, Millipore). All Alexa Fluor $^{\circledR}{ }^{\circledR}{ }^{-t a g g e d}$ secondary antibodies were sourced from Jacskon ImmunoResearch Europe Ltd

Microfluidic Devices: Cultured induced neurons were fixed in $4 \%$ paraformaldehyde in PBS for 15 min at room temperature, washed three times with PBS, and permeabilized with $0.3 \%$ Triton X-100 in PBS for 5 min at room temperature. Cells were blocked in PBS containing $5 \%$ normal donkey serum for $1 \mathrm{~h}$ at room temperature before overnight incubation at $4^{\circ} \mathrm{C}$ with the following primary antibodies: MAP2 (188006, Synaptic Systems); Homer 1 (160004, Synaptic Systems), Synaptophysin (101011, Synaptic Systems), and GFAP (AB5804, Millipore). Cells were washed twice with PBS and incubated with the following secondary antibodies for $2 \mathrm{~h}$ at RT: DyLight ${ }^{\mathrm{TM}} 405$ Donkey Anti-Chicken (703-475-155, Jackson ImmunoResearch), Alexa Fluor 594 Donkey Anti-Guinea Pig (706-585-148, Jackson ImmunoResearch), Alexa Fluor 488 Donkey Anti-Mouse (715-545-151, Jackson ImmunoResearch) and Alexa Fluor 647 Donkey Anti-Rabbit (711-605-152, Jackson ImmunoResearch). Cells were rinsed three times with PBS and microfluidic devices were mounted with $90 \%$ glycerol.

Samples were imaged with a LSM 880 confocal microscope with a 63X 1.4 NA objective. Images were acquired at zoom 2 in z-stacks of $0.5 \mu \mathrm{m}$ interval. Typically, 6 images were acquired per device from the synapse chamber near the postsynaptic chamber such the image contains multiple dendrites. Images were deconvoluted using the Huygens software (Scientific Volume Imaging, Netherlands).

Cerebral Organoids: Cerebral organoids were fixed in $4 \%$ PFA $(w / v)$ for 30 min at $4^{\circ} \mathrm{C}$ followed by three washes with PBS 0.1 M. Cerebral organoids were then placed in sucrose solution (30\% w/v) overnight before being embedded in O.C.T (Tissue-Tek). Embedded tissue was sectioned at $20 \mu \mathrm{m}$ using a Cryostar NX70 Cryostat (Thermo Scientific) and mounted slides were stored at $-80^{\circ} \mathrm{C}$ until immunostaining was performed. For immunostaining, tissue sections were brought to room temperature and then rehydrated with 3 washes with $0.1 \mathrm{M}$ PBS, each for 5 mins. Slides were then washed once with PBS with $0.2 \%$ Triton X-100 for 15 mins. Tissue was blocked using $10 \%$ of donkey serum in PBS $0.1 \mathrm{M}$ for $1 \mathrm{~h}$ at room temperature. After blocking, primary antibodies were added to $0.2 \%$ Triton X-100 and $10 \%$ of donkey serum in PBS $0.1 \mathrm{M}$ at appropriate dilutions and incubated overnight at $4^{\circ} \mathrm{C}$. The next day, slides were washed with PBS $0.1 \mathrm{M} 3$ times for 5 min each with gentle shaking. Subsequently, slides were incubated with Alexa Fluor $^{\circledR}$-conjugated secondary antibodies in 
$0.2 \%$ Triton $\mathrm{X}-100$ and $10 \%$ of donkey serum in PBS $0.1 \mathrm{M}$ for $2 \mathrm{~h}$ at room temperature in the dark. After secondary antibody incubation, slides were washed 3 times with PBS for 5 min with gentle shanking. Nuclei were visualized by incubating the tissue for 5 min with Hoechst 33258 stain in PBS 0.1 M. Sections were mounted using aqueous mounting medium (Polysciences). Images were acquired using an LSM 880 Confocal Scanning Microscope in concert with the ZEISS ZEN imaging software housed at the Imaging Platform of the Pasteur Institute, Lille. Image acquisition was done at 40X for the various cellular markers in Fig. 1. The antibodies used were MAP2 (188006, Synaptic Systems) and GFAP (AB5804, SigmaAldrich).

\section{Quantification of Synaptic Connectivity}

Synaptic connectivity was quantified as previously described (Kilinc et al, 2020). Briefly, images were analyzed with Imaris software (Bitplane, Zürich, Switzerland) by reconstructing Synaptophysin I and Homer puncta in 3D. The volume and position information of all puncta were processed using a custom Matlab (MathWorks, Natick, MA) program. This program assigns each postsynaptic spot to the nearest presynaptic spot (within a distance threshold of $1 \mu \mathrm{m})$ and calculates the number of such assignments for all presynaptic puncta.

\section{Immunoblotting}

Samples from the 2D cultures or brain organoids were collected in RIPA buffer containing protease inhibitors (Complete mini, Roche Applied Science) and sonicated two times at 60\% - 70\% for 10 seconds prior to use for the immunoblotting analyses.

Protein quantification was performed using the BCA protein assay (ThermoFisher Scientific). $10 \mu \mathrm{g}$ of protein from extracts were separated in 4-12\% SDS-polyacrylamide gels (NuPAGE Bis-Tris, Thermo Scientific) and transferred on to nitrocellulose membranes (BioRad). Next, membranes were incubated in milk ( $5 \%$ in Tris-buffered saline with $0.1 \%$ Tween20 (TBST) or SuperBlock (ThermoFisher Scientific) to block non-specific binding sites for 1 hour at room temperature, followed by several washes with TBST. Immunoblottings were carried out with primary antibodies overnight at $4^{\circ} \mathrm{C}$ under shaking condition. The membranes were washed three times in TBST, followed by incubation with HRP-conjugated secondary antibodies overnight at $4^{\circ} \mathrm{C}$ under shaking condition. The membranes were washed three times in TBST, and the immunoreactivity was revealed using the ECL chemiluminescence 
system (SuperSignal, Thermo Scientific) and imaged using the Amersham Imager 600 (GE Life Sciences). Optical densities of bands were quantified using the Gel Analyzer plugin in Fiji ImageJ.

The primary antibodies used for the immunoblots were as follows: BIN1 (ab182562, Abcam), APP C-terminal (A8717, Sigma-Aldrich), Tau (A002401-2, Agilent) Phospho-Tau (Clone: AT8) (MN1020, ThermoFisher Scientific), and B-ACTIN (A1978, Sigma-Aldrich). Secondary antibodies used for the immunoblots were Mouse-HRP (115-035-003, Jackson ImmunoResearch) and Rabbit-HRP (111-035-003, Jackson ImmunoResearch).

\section{AlphaLISA measurements}

Cell culture media samples for AlphaLISA measurements were collected at the endof the 3rd and 4th weeks of differentiation of the ASCL1-hiNs. Alpha-LISA kits specific for human $A \beta 1-X$ (AL288C, PerkinElmer) and AB1-42 (AL276C, PerkinElmer) were used to measure the amount of $A \beta 1-X$ and $A \beta 1-42$ respectively in culture media. The human $A \beta$ analyte standard was diluted in the BrainPhys medium. For the assay, $2 \mu \mathrm{L}$ of cell culture medium or standard solution was added to an Optiplate-384 microplate (PerkinElmer). $2 \mu \mathrm{L}$ of $10 \mathrm{X}$ mixture including acceptor beads and biotinylated antibody was then added to the wells with culture media or standard solution. Following incubation at room temperature for an hour, $16 \mu \mathrm{L}$ of $1.25 \mathrm{X}$ donor beads was added to respective wells and incubated at room temperature for 1 hour. Luminescence was measured using an EnVision-Alpha Reader (PerkinElmer) at 680-nm excitation and 615-nm emission wavelengths.

\section{Calcium and iGluSnFR Imaging}

Calcium imaging was performed in 2D cultures after 4-weeks (Ascl1-induced). Prior to imaging, the cells were incubated with Oregon Green ${ }^{\mathrm{TM}} 488$ BAPTA-1 (OGB-1) acetoxymethyl (AM) (ThermoFisher Scientific) for 1 hour. A $2.5 \mathrm{mM}$ stock solution of the calcium-indicator dye was prepared in Pluronic ${ }^{\mathrm{TM}} \mathrm{F}-127$ (20\% solution in DMSO) (ThermoFisher Scientific). $1 \mu \mathrm{L}$ of the dye solution was added to $400 \mu \mathrm{L}$ of fresh BrainPhys medium in each well of a 24-well cell imaging plate. Existing BrainPhys media from the wells of the plate was removed and kept aside while the calcium-indicator dye was incubated in fresh BrainPhys medium. After the 1hour incubation, the medium which was kept aside was replaced to each well. The 2D cultures 
were then ready to be filmed using a Spinning Disk Microscope housed at the Institut Pasteur de Lille, Lille, France using the MetaMorph imaging software.

For filming the calcium activity, 1000 images were taken using a 20X long-distance objective, $10 \mathrm{~ms}$ exposure time and $200 \mathrm{~ms}$ intervals. For each well, 5 random fields were chosen, and the cellular activity was, thus, recorded.

For treatments with pharmacological agents - Bicuculline $(100 \mu \mathrm{M})$ and CNQX $(100 \mu \mathrm{M})$, the recordings were done in two steps. For each well, 5 random fields were chosen to record cell activity. The spatial coordinates of these fields were saved on the imaging software. Drug application was done, and the imaging plate was allowed to stand for a period of 5 minutes. The well to which drug was applied was then subsequently filmed at the pre-saved coordinates on the imaging software, thereby ensuring that the same fields were recorded for both pre- and post-drug treatment conditions.

For cells transduced with iGluSnFR, these cells were directly filmed after 4 weeks of differentiation and 500 images were taken using a 20X long-distance objective, $10 \mathrm{~ms}$ exposure time and $200 \mathrm{~ms}$ intervals. Up to 8 fields per well were filmed, each field containing at least one fluorescent transduced cell along with its processes.

\section{Analyses of Calcium Transients}

All live recordings of neuronal calcium transients were first converted into .avi format after background subtraction using the FIJI software. Following these, the videos were subsequently opened using the free software for data analyses of calcium imaging, CALciumIMagingAnalyzer (CALIMA) made available online by Fer Radstake (Eindhoven University of Technology, The Netherlands). Each video recording of a field of cells was first downscaled to $2 \mathrm{X}$ in terms of size with a $10 \mathrm{X}$ zoom and was checked for the frame average mode. Moreover, in this first detection stage, pre-set filter parameters were adjusted and applied to enable the detection of the maximum number of fluorescent cells in each field. In the analysis tab, detection of the average activity was checked and for pre-processing, a median of 3 was applied. All cells within the pre-set filter parameters are detected as regions of interest (ROIs) in the detection stage. Cell activity from all detected ROIs is then recorded. However, in the subsequent analysis stage, only cells showing spiking frequencies with a standard deviation of at least 2 or more were taken into consideration. Data in the form of 
detection spikes and the correlation (peak) are extracted and exported as CSV files. All statistical analyses of extracted data were performed on GraphPad Prism Software.

\section{Electrophysiological recordings in 2D cultures and cerebral organoids}

ASCL1-hiNs were cultured in microfluidics devices bound to multi-electrode arrays (256MEA30/8iR-ITO, Multi Channel Systems, Germany) devices as described above. Extracellular action potentials were recorded in 5 different cultures of both genotypes at 2, 3, 4 and 6 weeks of differentiation using the MEA2100-256-System (Multi Channel Systems, Germany). Before recordings, MEAs were left immobile for $5 \mathrm{~min}$ in the head stage, to reduce artifacts due to medium movement. Signals were recorded for $1 \mathrm{~min}$, at $40 \mathrm{kHz}$ sampling rate, using the software Multi Channel Experimenter 2.16.0. Transient potentials (multiunit activity - MUA) were detected and extracted using 5.5 standard deviations upward or downward of the raw signal and a dead zone of $3 \mathrm{~ms}$. Quantification of the number of detected spikes (MUAs) and spike bursts (defined as at least 5 spikes within $50 \mathrm{~ms}$ ) was performed using the software Multi Channel Analyzer 2.16.0.

Electrical activity in cerebral organoids was recorded using 256-6wellMEA200/30iR-ITO (Multi Channel Systems, Germany). Briefly, 5-6 months old cerebral organoids were mounted onto MEAs and kept for $2 \mathrm{~h}$ in complete Brainphys medium. Then, MEAs were placed in the head stage and left still for 5 min before recordings. Signals were recorded for $5 \mathrm{~min}$, at 10 $\mathrm{kHz}$ sampling rate using the software Multi Channel Experimenter 2.16. Transient potentials (multiunit activity - MUA) were detected and extracted using 4.5 standard deviations upward or downward of the raw signal and a dead zone of $3 \mathrm{~ms}$. Quantification of the number of detected spikes (MUAs) and spike bursts (defined as at least 5 spikes within $50 \mathrm{~ms}$ ) was performed using the software Multi Channel Analyzer 2.16.0.

\section{Spike sorting}

Channels containing putative waveforms were manually processed offline for spike waveform separation and classification using Offline Sorter v3 (Plexon, USA). Briefly, we applied principal component analysis (PCA) to the spike waveforms to cluster and separate waveforms of similar morphologies. Using this approach, we identified 2 to 10 well-isolated units per channel, and therefore, we considered this single-unit activity (SUA). For each SUA, we computed the average firing rate, the signal-to-noise ratio, the peak-to-trough amplitude 
and duration, the average power (square amplitude of the average waveform), the mode of the interspike interval distribution, and their firing patterns. It has been demonstrated that dissociated neuronal cultures can develop complex discharge structures (Wagenaar, 2006). Here, we considered burst activity if the SUA presents periods of high-frequency discharges interspersed by regular or no discharges at all. Operationally, a burst must have at least 3 discharges within $100 \mathrm{~ms}$ and $200 \mathrm{~ms}$ intervals, for the interval between the first and the second, and the second and the third discharge, respectively. After the third spike, the maximal interval to consider a discharge part of the burst was $200 \mathrm{~ms}$. Thus, we computed the SUA that presented bursts, the number of bursts (i.e., the burst frequency), the average burst duration, the number of spikes within each burst, the average burst frequency, and the inter-burst interval.

\section{snRNA-seq Library Preparation}

Nuclei isolation and Hash-tagging with oligonucleotides steps were realized on ice with pre-cold buffers and centrifugations at $4^{\circ} \mathrm{C} .6 .5$ months-old BIN1 WT, HET, and KO organoids were processed as previously (Lambert et al., 2022). 4 weeks-old cultured ASCL1-induced BIN1 WT and KO 2D cultures were washed in the imaging plate wells with $500 \mu \mathrm{L}$ of Deionized Phosphate Buffer Saline 1X (DPBS, GIBCO ${ }^{\mathrm{TM}}$, Fisher Scientific 11590476). Cells were resuspended with wide bore tips in $500 \mu \mathrm{L}$ Lysis Buffer (Tris- $\mathrm{HCL} 10 \mathrm{mM}, \mathrm{NaCl} 10 \mathrm{mM}, \mathrm{MgCl} 2$ 3mM, Tween-20 0,1\%, Nonidet P40 Substitute 0,1\%, Digitonin 0,01\%, BSA 1\%, Invitrogen ${ }^{\mathrm{TM}}$ RNAseout $^{\mathrm{TM}}$ recombinant ribonuclease inhibitor $\left.0,04 \mathrm{U} / \mu \mathrm{L}\right)$. Multiple mechanical resuspensions in this buffer were performed for a total lysis time of 15 mins., $500 \mu \mathrm{L}$ of washing buffer was added (Tris-HCL 10mM, NaCl 10 mM, MgCl2 3 mM, Tween-20 0.1\%, BSA $1 \%$, Invitrogen ${ }^{\mathrm{TM}}$ RNAseout $^{\mathrm{TM}}$ recombinant ribonuclease inhibitor $0,04 \mathrm{U} / \mu \mathrm{L}$ ) and the lysis suspension was centrifuged 8 mins. at $500 \mathrm{~g}$ (used for all following centrifugation steps). Nuclei pellets were washed tree times with one filtration step by MACS pre-separation filter $20 \mu \mathrm{m}$ (Miltenyi Biotec). Nuclei pellets were resuspended in $100 \mu \mathrm{L}$ of staining buffer (DPBS BSA 2\%, Tween-20 0.01\%), $10 \mu \mathrm{L}$ of Fc blocking reagent HumanTruStainFc ${ }^{\mathrm{TM}}$ (422302, Biolegend) and incubated $5 \mathrm{~min}$ at $4^{\circ} \mathrm{C}$. $1 \mu$ lof antibody was added (Total-Seq ${ }^{\mathrm{TM}}-\mathrm{A} 0453$ antiVertebrate Nuclear Hashtag 3 MAb414 for the WT and Total-Seq ${ }^{\mathrm{TM}}-\mathrm{A} 0454$ anti-Vertebrate Nuclear Hashtag 4 MAb414 for the KO, 97286 and 97287 respectively, Biolegend) and incubated 15 mins. at $4^{\circ} \mathrm{C}$. Nuclei pellets were washed three times in staining buffer with one 
filtration step by MACS pre-separation filter $20 \mu \mathrm{m}$ (Miltenyi Biotec) to a final resuspension in $300 \mu \mathrm{L}$ of staining buffer for Malassez cell counting with Trypan blue counterstaining (Trypan Blue solution, 11538886, Fisherscientific). Isolated nuclei were loaded on a Chromium 10X genomics controller following the manufacturer protocol using the chromium single-cell v3 chemistry and single indexing and the adapted protocol by Biolegend for the HTO library preparation. The resulting libraries were pooled at equimolar proportions with a 9 for 1 ratio for Gene expression library and HTO library respectively. Finally, the pool was sequenced using 100pb paired-end reads on NOVAseq 6000 system following the manufacturer recommendations (Illumina).

snRNA-seq Dataset Preprocessing

Unique Molecular Index (UMI) Count Matrices for gene expression and for Hash Tag Oligonucleotide (HTO) libraries were generated using the CellRanger count (Feature Barcode) pipeline. Reads were aligned on the GRCh38-3.0.0 transcriptome reference (10x Genomics). Filtering for low quality cells according to the number of RNA, genes detected, and percentage of mitochondrial RNA was performed. For HTO sample, the HTO matrix was normalized using centered log-ratio (CLR) transformation and cells were assigned back to their sample of origin using HTODemux function of the Seurat R Package (v4)[10]. Then, normalizations of the gene expression matrix for cellular sequencing depth, mitochondrial percentage and cell cycle phases using the variance stabilizing transformation (vst) based Seurat:SCTransform function were performed.

\section{SnRNA-seq datasets integration and annotation}

To integrate the datasets from independent experiments, the harmony $R$ package (https://github.com/immunogenomics/harmony) was used. In order to integrate the datasets, the SCTransform normalized matrices was merged and PCA was performed using Seurat::RunPCA default parameter. The 50 principal components (dimensions) of the PCA were corrected for batch effect using harmony::RunHarmony function. Then, the 30 first batch corrected dimensions were used as input for graph-based cell clustering and visualization tool. Seurat::FindNeighbors using default parameters and Seurat::FindClusters function using the Louvain algorithm were used to cluster cells according to their batch corrected transcriptomes similarities. To visualize the cells similarities in a 2-dimension space, 
the Seurat::RunUMAP function using default parameter was used. Cell clusters were then annotated based on cell type specific gene expression markers.

\section{Differential gene expression and GO enrichment analyses}

Gene expression within each main cell type was compared between conditions of interest using Wilcoxon test on the SCTransform normalized gene expression matrix. GO enrichment analysis on the differentially expressed genes was performed using the gost function of the gprofiler2 R package (CRAN).

\section{Activity-related genes (ARGs) signature enrichment analysis at single cell resolution}

To study enrichment for activity-related genes (ARGs) signature across cerebral organoid cells, the CellID R package (https://github.com/RausellLab/CelliD) was used. ARGs obtained from Tyssowski et al. (2018) and Hravtin et al. (2018) (supplementary Table 7), were translated to the corresponding human gene name with the help of the biomaRt package using the respective Ensembl references. Then, the CellID::RunMCA was used to extract cellspecific gene signature and hypergeometric test was performed to test enrichment for ARGs in these cell signatures. To test the differential proportion of ARGs enriched cells in BIN1 deleted organoid compared to WT organoid, chi-squared test was performed.

\section{Comparative analysis with specific DEGs in AD brains}

To compare the transcriptomic change observed in BIN1 deleted cerebral organoid with those observed in AD brain, datasets from the work of Leng et al. (ref) and Morabito et al. (ref) were taken as 2 independent references. The raw gene expression matrix was normalized using Seurat::SCTransform and differential expression analysis was performed within each neuronal cell type using Wilcoxon test as used for our organoid dataset. AD related DEGs, thus, obtained were compared with our BIN1 related organoid DEGs in every cell type. To this end, the enrichment for AD-related DEGs in BIN1-related DEGs was tested using hypergeometric test. The background for this test was defined as all genes detected in both datasets. The $p$-value of this test was used as metrics to compare the significance of the gene overlap between neuronal cell types. 
Figure 1

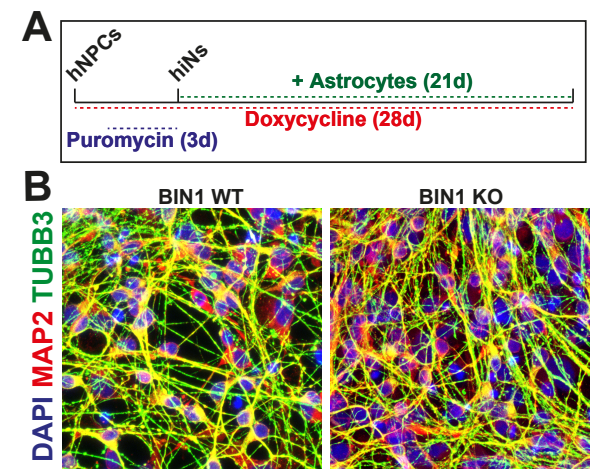

E

\section{Glutamatergic Neurons}

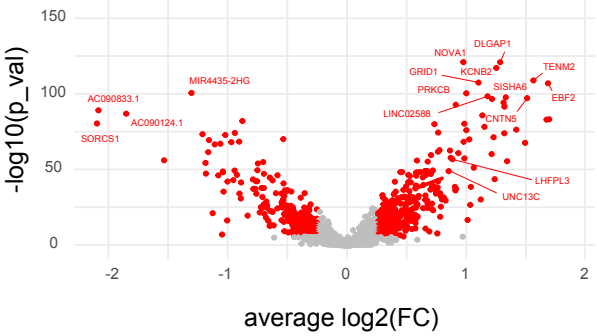

C

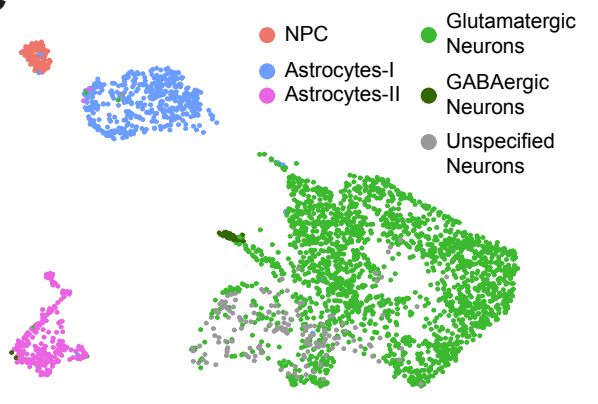

D

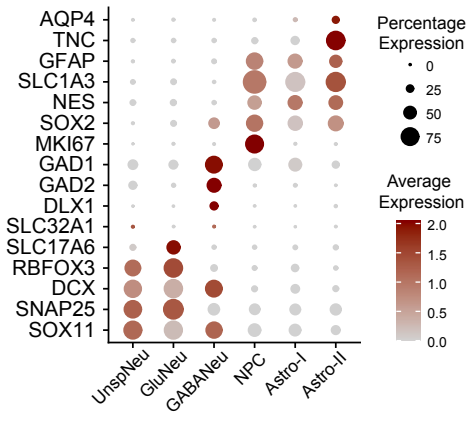

F

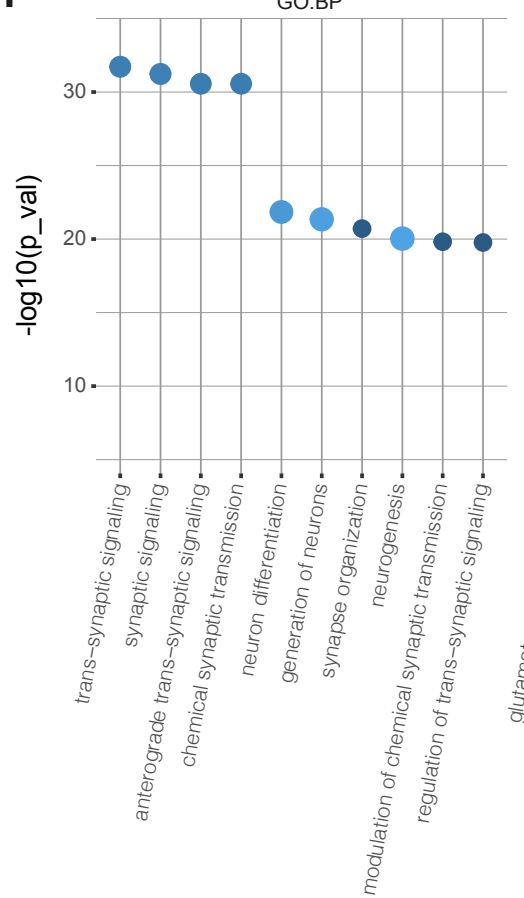

GO:MF

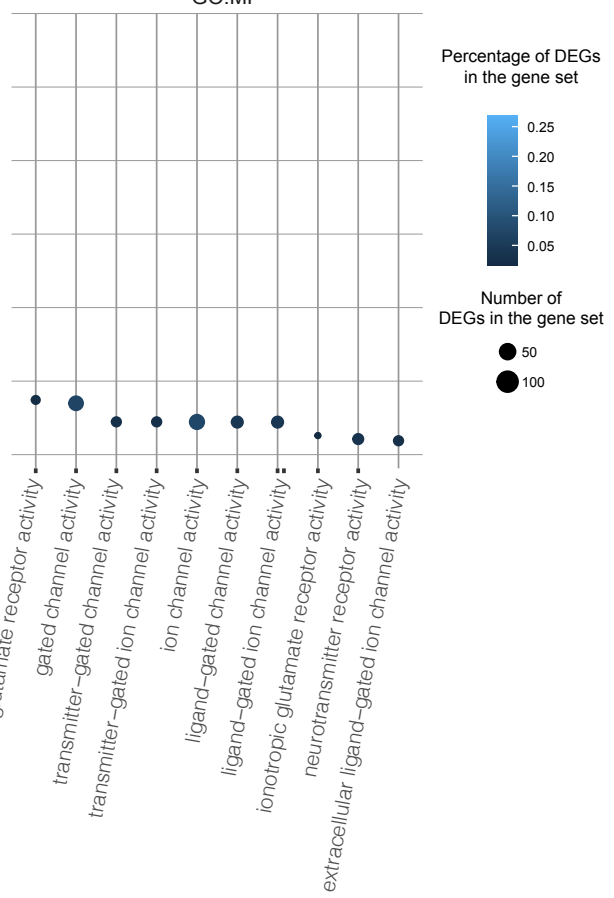


A

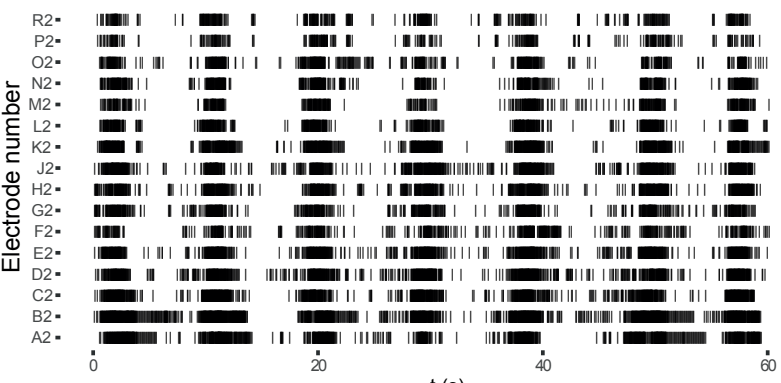

$\mathrm{t}(\mathrm{s})$

B

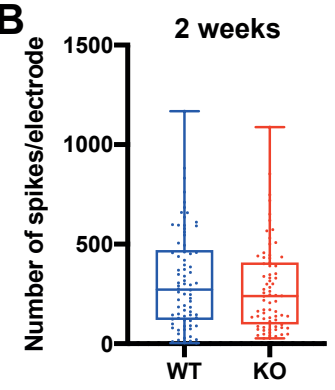

응
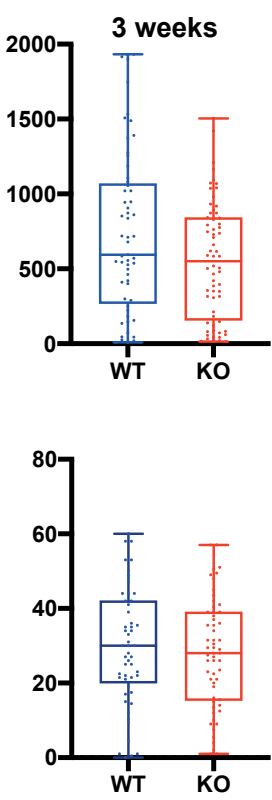

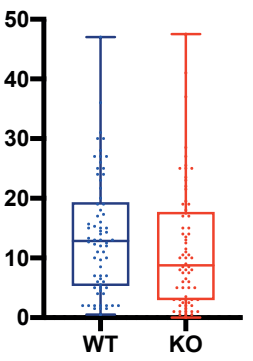

BIN1 KO ASCL1-hiNs 4 weeks

R2. || |||

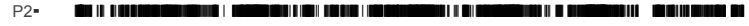

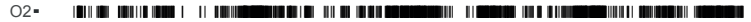

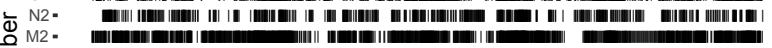
을 $\mathrm{M} 2$

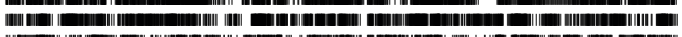

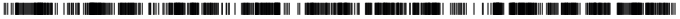

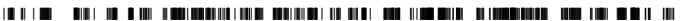

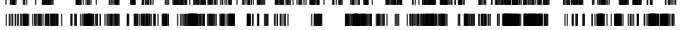

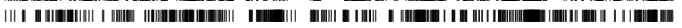

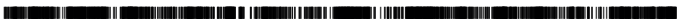

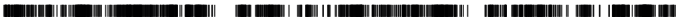

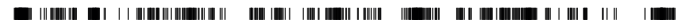

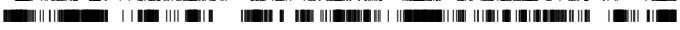

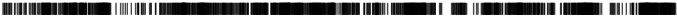
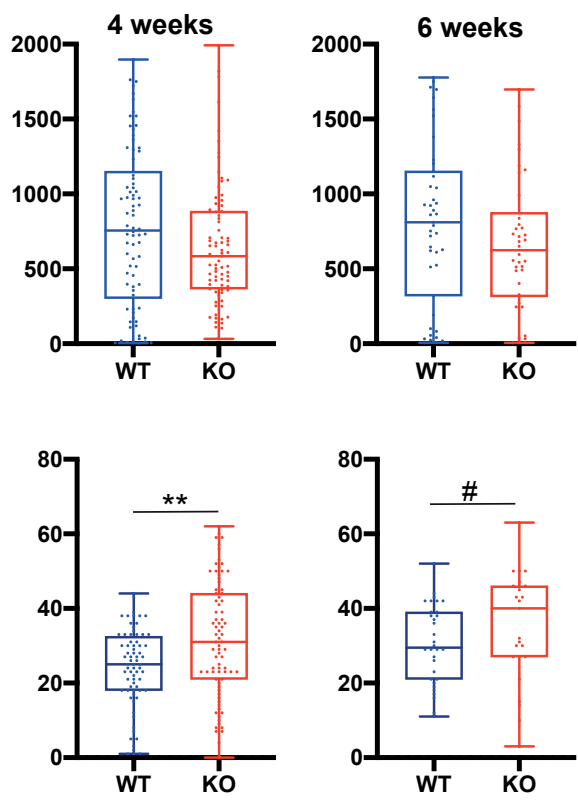
$\dot{0}$

20

'ं0

$\mathrm{t}(\mathrm{s})$

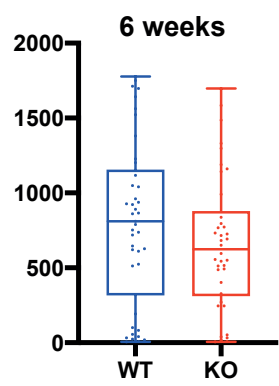

C

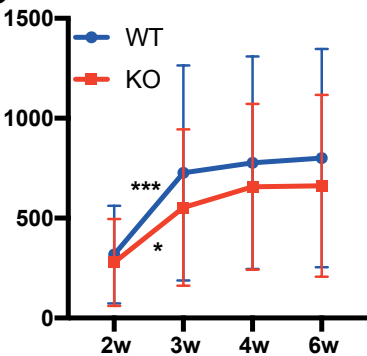


A multi-unit activity

- 1

spike waveform clustering

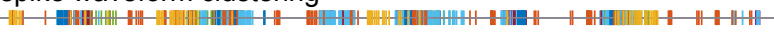

sorted spike waveforms / single-unit activity

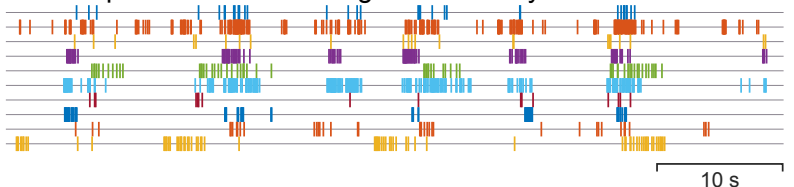

D WT

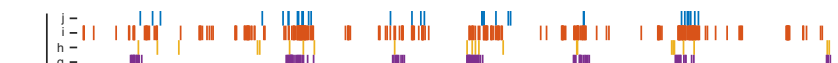

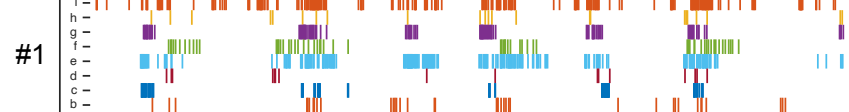

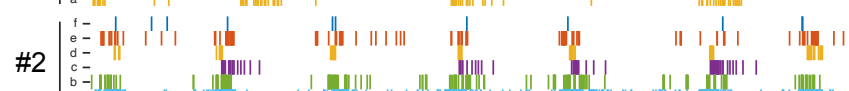

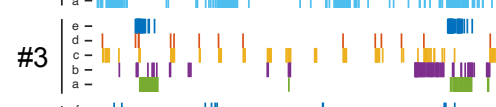

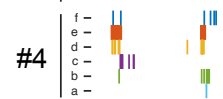

III

$\left.\right|_{\| 1} ^{1}=$

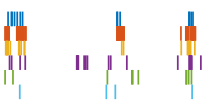

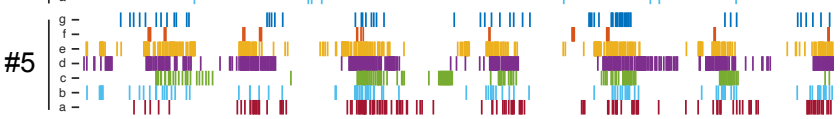

$\mathbf{E}$

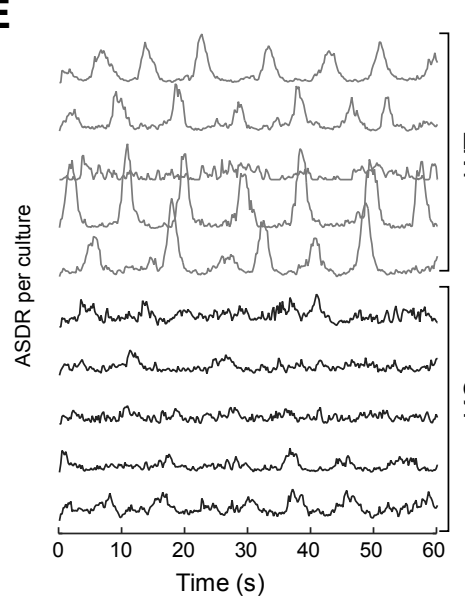

B

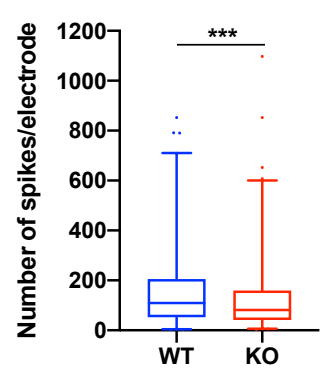

$\mathrm{KO}$

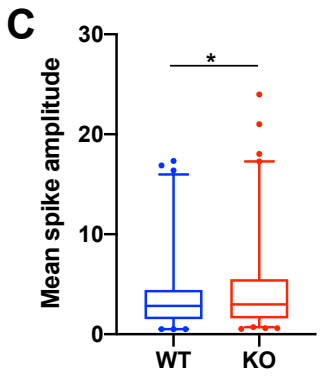

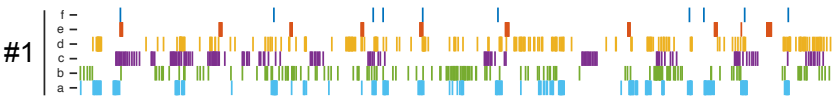

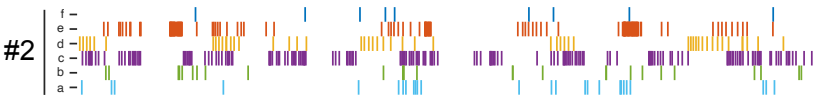

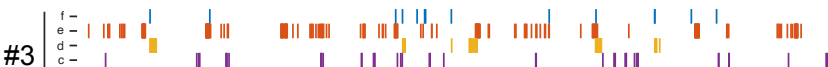

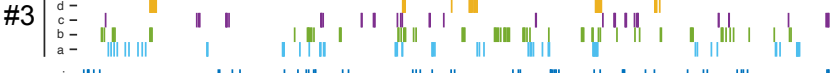

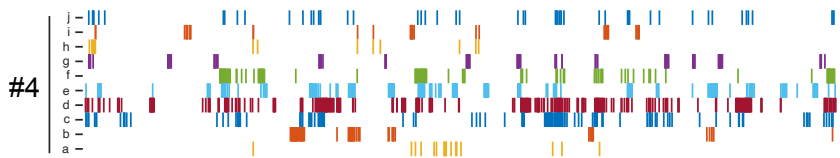

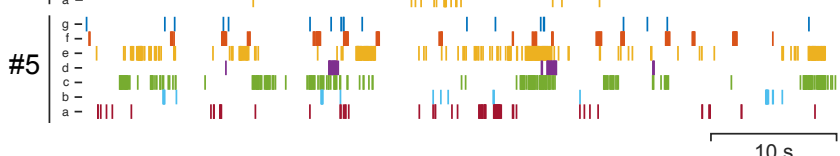

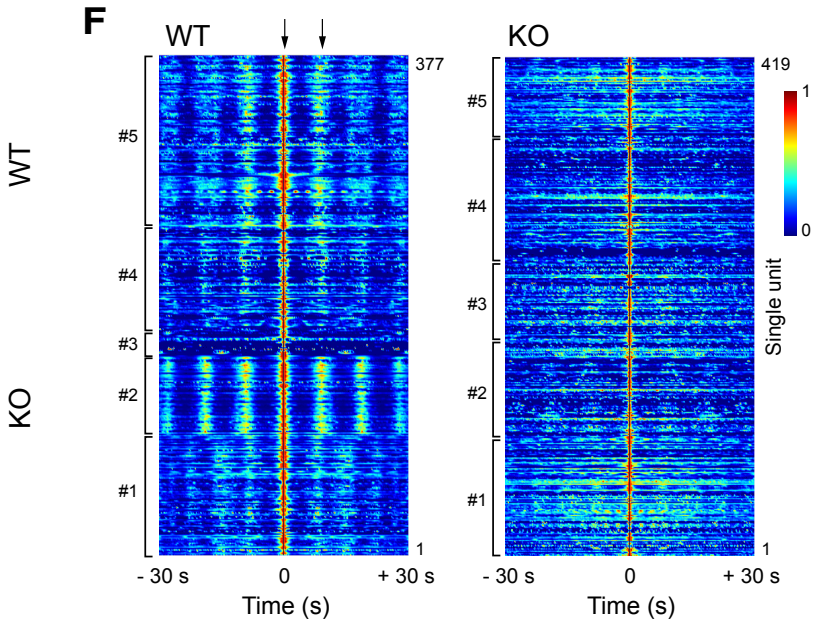

$\mathbf{G}$

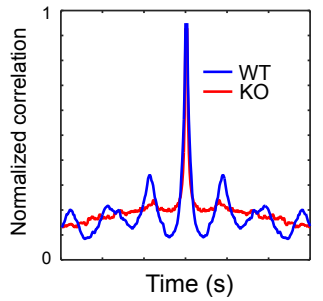




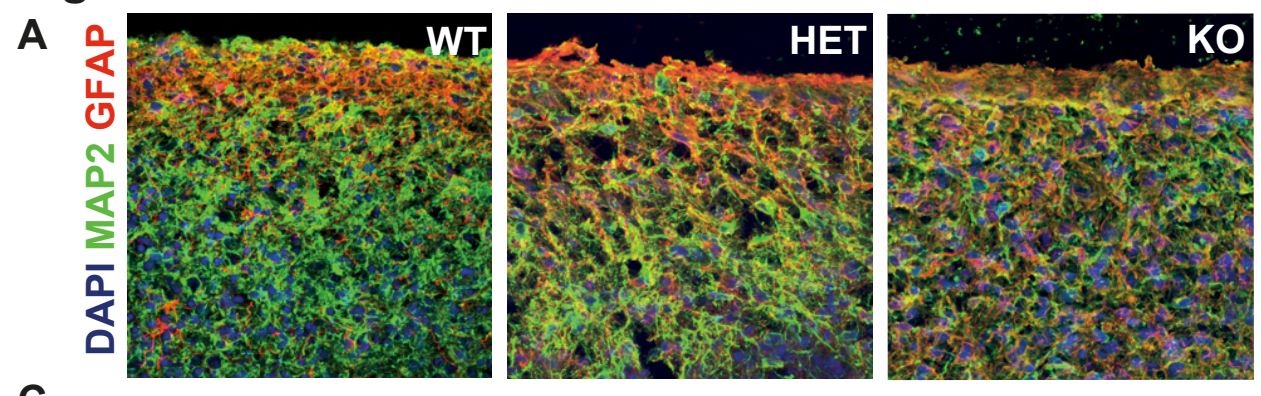

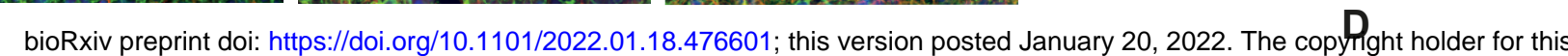

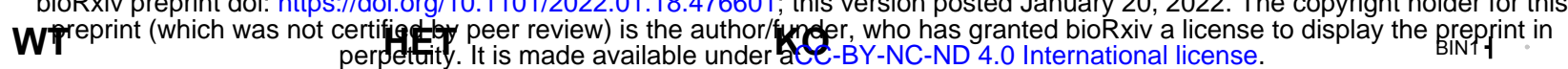

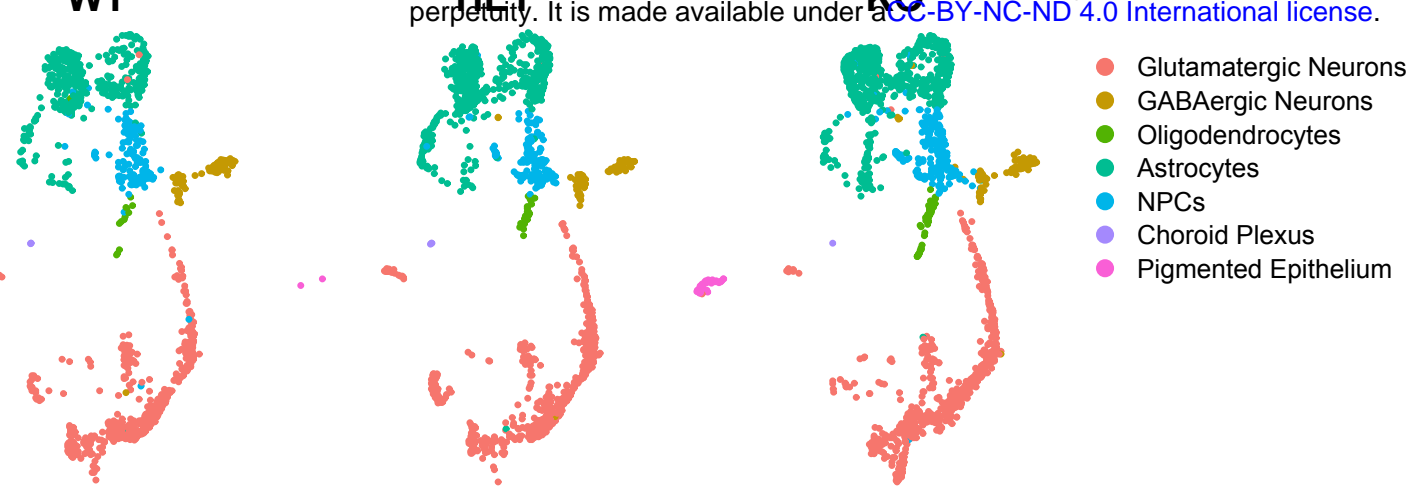

Astrocytes

Glutamatergic Neurons

80

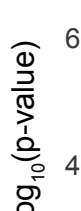

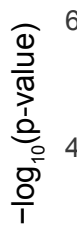

20

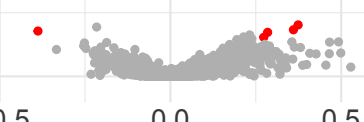
PKIB

G
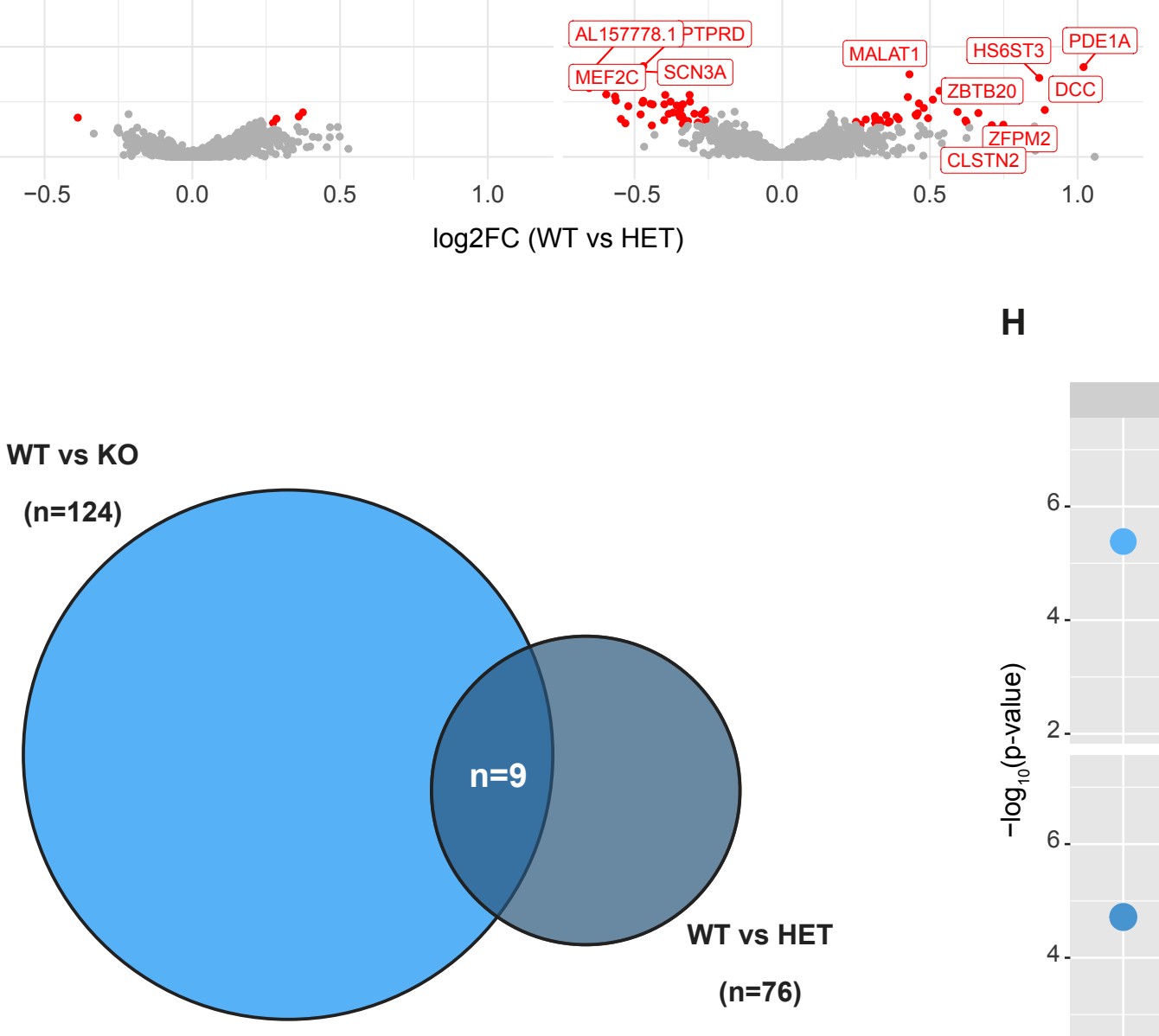

H
B WT WT WT HET HET HET KO KO $\mathrm{kDa}$ BIN1

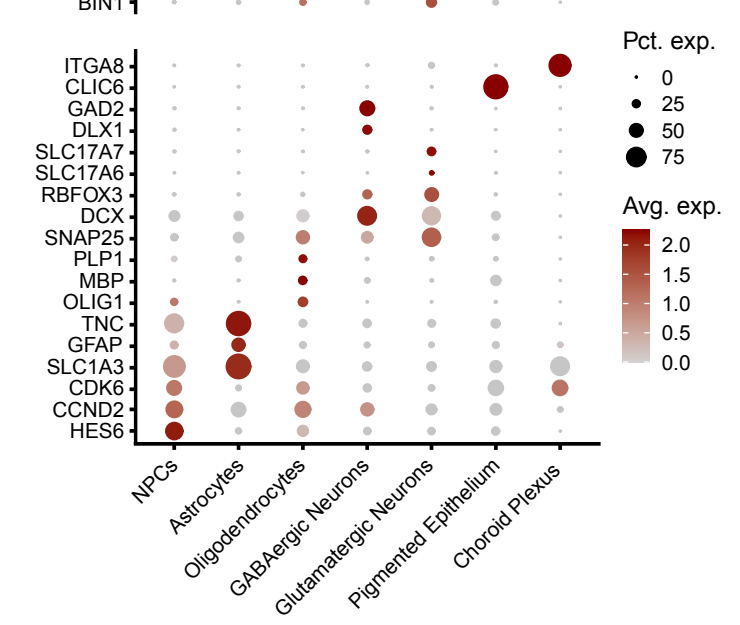

E

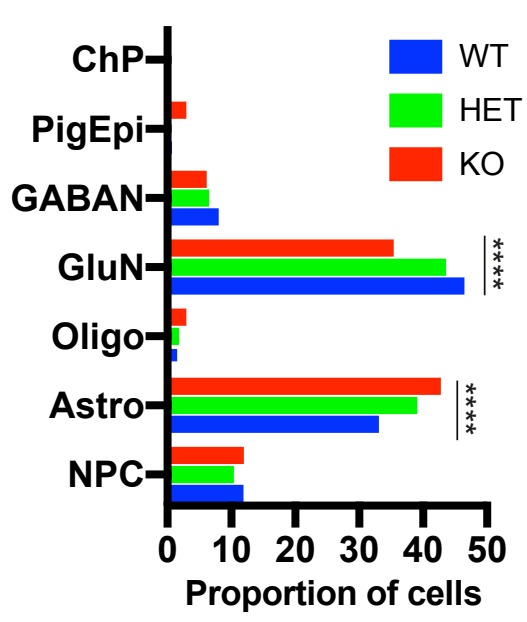

Astrocytes

Glutamatergic Neurons

PKIB].

MALAT1

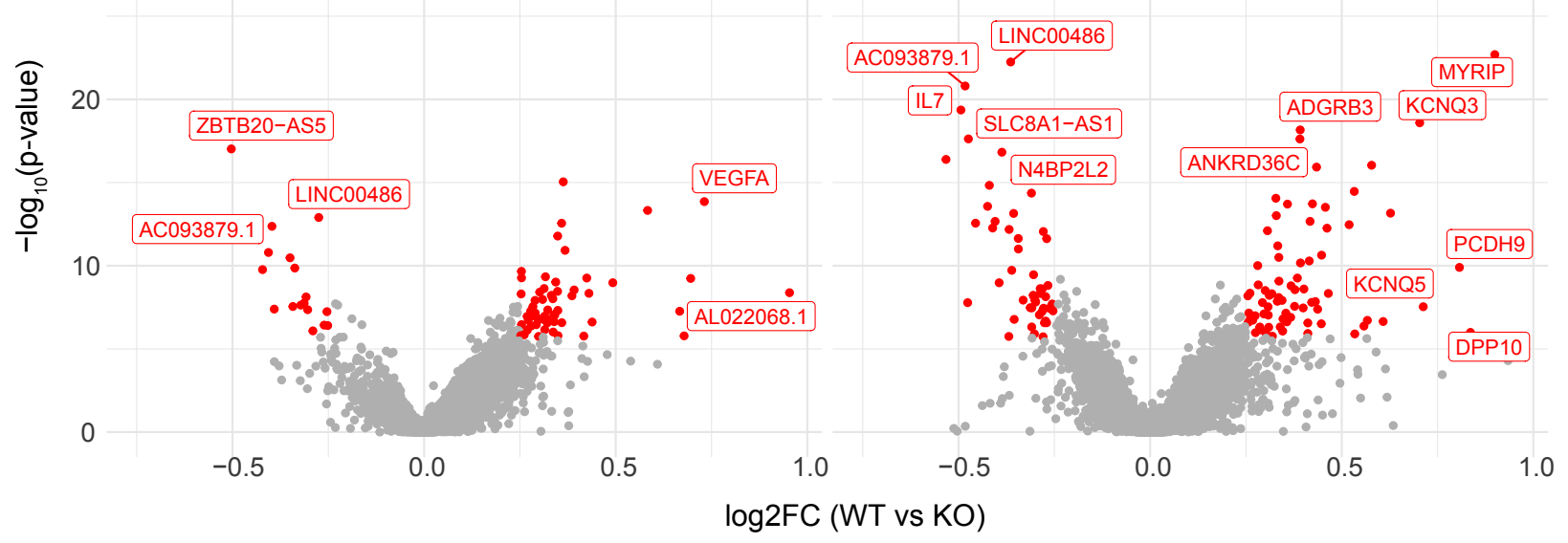

DEG
adjusted $p$-value $<0.05$ $\left|\log _{2} \mathrm{FC}\right|>0.25$

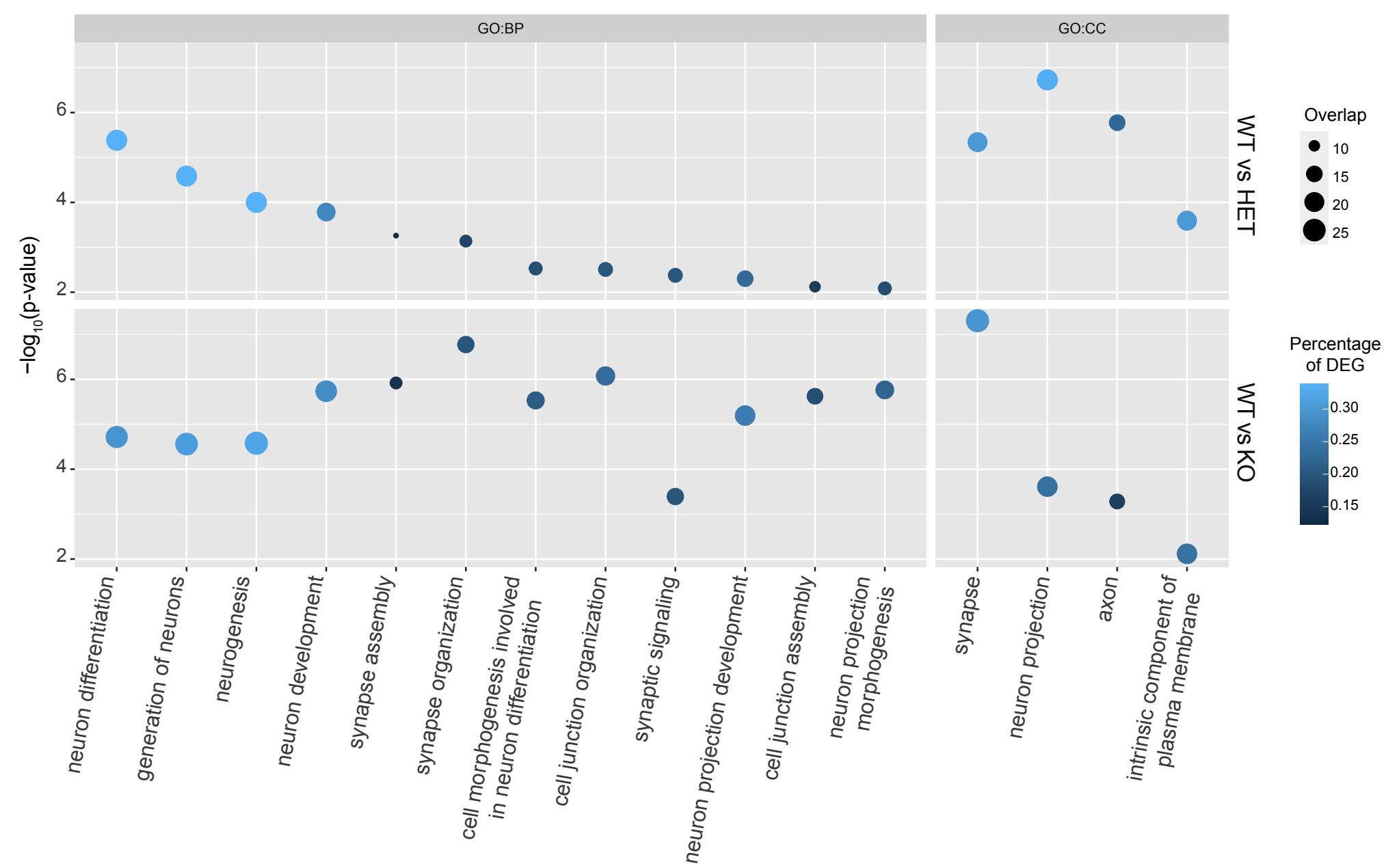


A

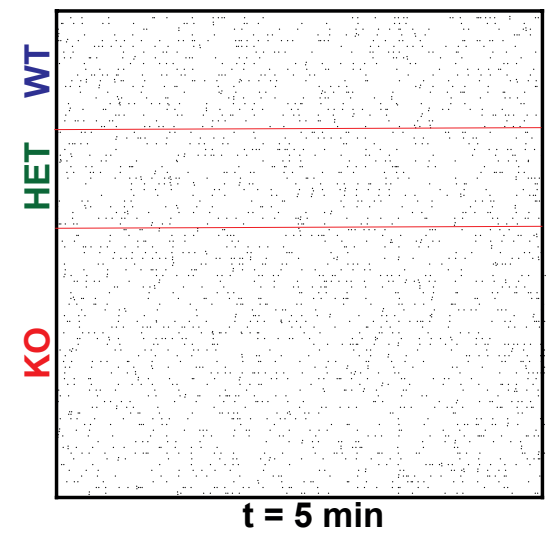

D

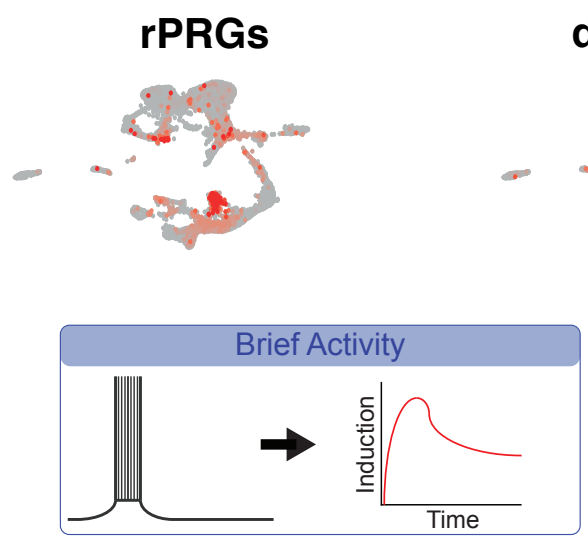

Glutamatergic Neurons

15

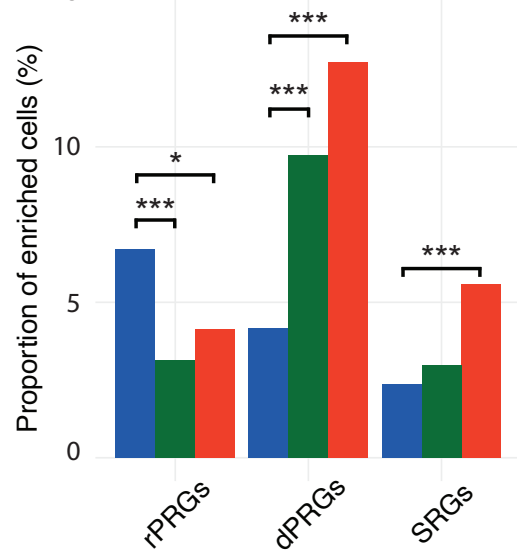

E

(1)

B

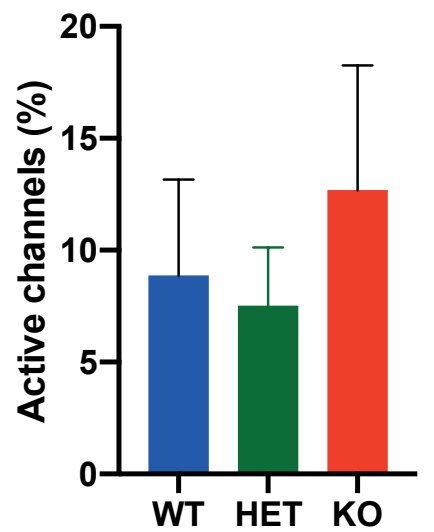

dPRGs

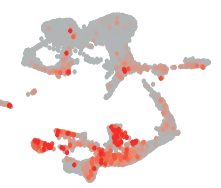

C

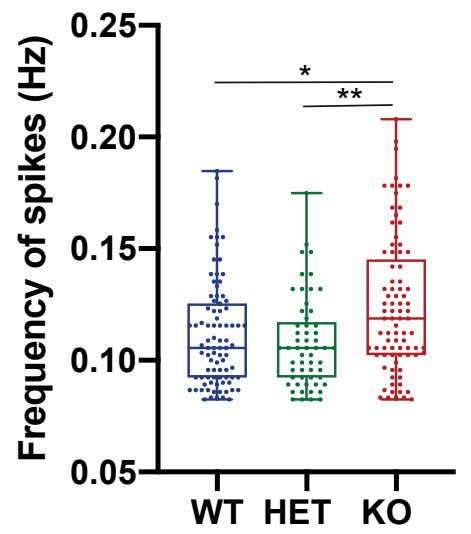

\section{SRGs}

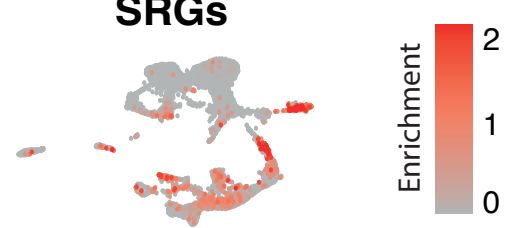

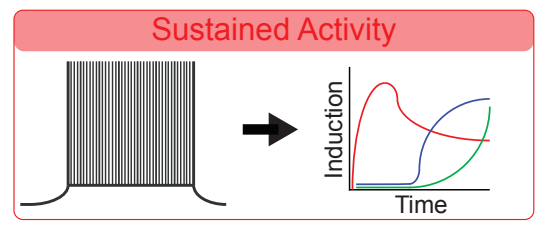

rPRGS

dPRGS

SRGS

GABAergic Neurons
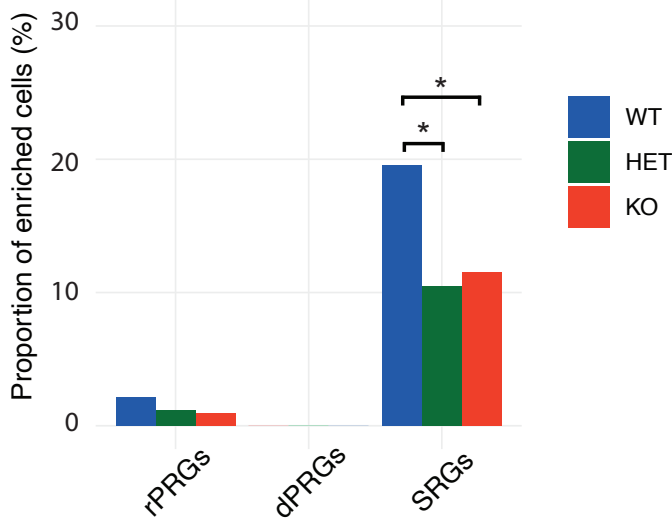

$s^{\mathrm{PO}^{5}}$ 
